UCRL-ID-120238

\title{
Final Report on the LLNL Compact Torus Acceleration Project
}

\author{
J. Eddleman, J. Hammer, C. Hartman, \\ H. McLean, and A. Molvik
}

March 19, 1995

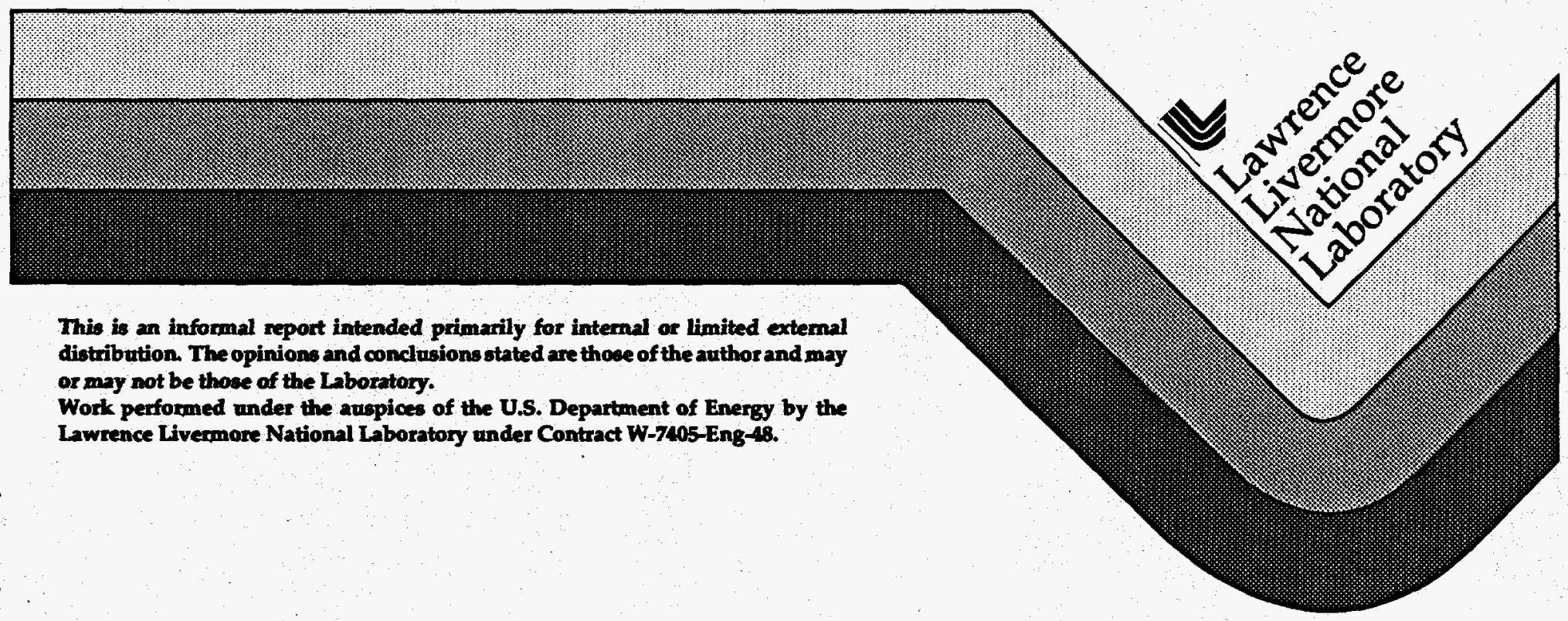


This document was prepared as an account of work sponsored by an agency of the United States Government. Neither the United States Government nor the University of California nor any of their employees, makes any warranty, express or implied, or assumes any legal liability or responsibility for the accuracy, completeness, or usefulness of any information, apparatus, product, or process disclosed, or represents that its use would not infringe privately owned rights. Reference herein to any specific commercial product, process, or service by trade name, trademark, manufacturer, or otherwise, does not necessarily constitute or imply its endorsement, recommendation, or favoring by the United States Government or the University of California. The views and opinions of authors expressed herein do not necessarily state or reflect those of the United States Government or the University of California, and shall not be used for advertising or product endorsement purposes.

This report has been reproduced directly from the best available copy.

Available to DOE and DOE contractors firom the Office of Scientific and Technical Information P.O. Box 62, Oak Ridge, TN 37831

Prices available from (615) 576-8401, FTS 626-8401

Available to the public from the National Technical information Service

U.S. Department of Commerce 5285 Port Royal Rd. Springfield, VA 22161 


\section{DISCLAIMER}

Portions of this document may be illegible in electronic image products. Images are produced from the best available original document. 


\section{Final Report on the LLNL Compact Torus Acceleration Project}

March 19, 1995

James Eddleman, James Hammer, Charles Hartman, Harry McLean, and Arthur Molvik

\section{Introduction}

In this report, we summarize recent work at LLNL on the compact torus (CT) acceleration project. The CT accelerator is a novel technique for projecting plasmas to high velocities and reaching high energy density states. The accelerator exploits magnetic confinement in the CT to stably transport plasma over large distances and to directed kinetic energies large in comparison with the CT internal and magnetic energy. Applications range from heating and fueling magnetic fusion devices, generation of intense pulses of $\mathrm{x}$-rays or neutrons for weapons effects and high energy-density fusion concepts.

Beginning in 1986, we conducted experiments with the Ring ACcelerator Experiment (RACE) accelerator at LLNL to establish proof-of-principal for the concept. The accelerator concept and early modeling studies are found in references 1 and 2 . The first experiments on CT acceleration are described in reference 3 and experiments on slow formation and compression in references 4 and 5. Applications are discussed in references 2, and 6-8. More recent RACE experiments that demonstrated the predicted feature of magnetic flux compression during CT focusing are described in section 1 below. The last experimental series from 1992 to 1993 studied the effects of drag on the CT from wall-evolved gases and potential ways of reducing the drag through heating the electrodes. Drag effects strongly limited the operating regime in the precompressor/accelerationfocusing geometry. Recent analysis of data from these experiments shows that the drag was primarily due to snowplow of the CT through gas filling the accelerator tube. The gas was apparently produced during the formation stage of the CT. These results are reported in section 2.1. Modeling of wall plasma effects is described in section 2.2.

From 1993 to 1994, we collaborated with the Air Force Phillips Laboratory on the MARAUDER device ${ }^{9}$ in a program of accelerator studies, improvements and CT stagnation experiments at higher energy than available on RACE. Experimental results and modeling of MARAUDER are summarized in section 3. Finally, we describe two recently-conceived variants of the accelerator concept that may offer advantages for some applications, including a low-voltage driver scheme 
in section 4.1, and a radial/ non-self similar accelerator in section 4.2. We conclude with a discussion of possible techniques for extending the operating regime of the $\mathrm{CT}$ accelerator to higher energies and energy densities.

\section{Focusing and Flux Compression Experiments on RACE}

In this section we report the three-fold compression in radius and length and five-fold increase in the magnetic field of a CT in the $7.4 \mathrm{~m}$-long coaxial-cone geometry in RACE. This compression follows a two-fold quasistatic compression, reported previously ${ }^{4,5}$, for a six-fold total compression. A CT, shown in Fig. 1.1, is a toroidal, or doughnut-shaped, plasma configuration similar to a tokamak plasma. The plasma in a CT is an electrically conducting, ionized gas heated to a few tens of $\mathrm{eV}$. A magnetic field is produced by currents flowing in the plasma and forms a set of nested surfaces to confine the particles, so a. CT can be used as a container to hold plasma during acceleration.

Compression of magnetically confined plasmas has generally been used for the purposes of increasing the plasma temperature and density by compressing in a time short compared with an energy or density confinement time. The experiments described here differ in that increasing the thermal energy of the plasma is not necessarily desireable, instead our goal is to increase the velocity and kinetic energy of a compact torus and compress it to a small cross section and short length so as to deliver a higher power density in a shorter time. This is an alternative to the electrical pulse compression techniques usually used in pulsed power.

We calculate the scaling of the poloidal magnetic field $B_{p}$ with the plasma major radius $R$ assuming that poloidal flux, $\Psi_{p}$, is conserved. This can be written as

$$
\Psi_{p}=\int B_{p} d A=\text { const. }
$$

where $A$ is the area of the poloidal cross section. $A$ is approximately the product of $\Delta_{p}$, the thickness of the flux layer, and $2 \pi R$, the circumference of the outer electrode. For a CT in axial force balance, $\Delta_{p}$ is proportional to $\Delta$, the ? $p$ between the inner and outer coaxial electrodes. $\Delta$ is proportional to $R$ for the sei-imilar

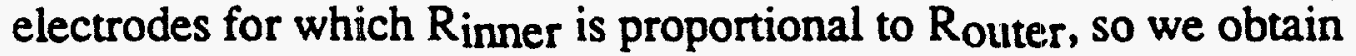

$$
\Psi_{p}=2 \pi R \Delta_{p} B_{p} \sim R^{2} B_{p}
$$

from which the scaling of $B_{p}$ is apparent

$$
B_{p}-\Psi_{p} / R^{2}
$$


If toroidal flux is also conserved, i.e.

$$
\Psi_{\mathrm{T}} \sim \mathrm{B}_{\mathrm{T}} \mathrm{L}\left(\Delta-2 \Delta_{\mathrm{P}}\right) \sim \mathrm{B}_{\mathrm{T}} \mathrm{LR}
$$

where $L$ is the length, then

$$
\mathrm{B}_{\mathrm{T}} \sim \Psi_{\mathrm{T}} / \mathrm{LR} \text {. }
$$

In magnetic pressure balance, $B_{T}{ }^{2}=B_{p}{ }^{2}$, and if $B<<1$, as expected for plasma near the Taylor minimum energy state, then we have

$$
\mathrm{L} \sim \mathrm{R} \sim \Delta .
$$

That is, when forced to compress self similarly in radius, the compact torus also compresses self similarly in length.

In previous experiments, we demonstrated CT formation and precompression as well as acceleration in straight coaxial electrodes. Some of the earlier straight acceleration experiments, without a precompression cone, were followed by a focusing cone. Resilient, stable magnetically-confined rings were formed and accelerated to velocities as high as $3 \times 10^{8} \mathrm{~cm} / \mathrm{s}$ in the straight system. Focusing, or compression, to smaller radii was observed when a CT entered a cone at high velocity, but the CT magnetic field did not scale as $R^{-2}$. If the cone were too short, with too steep an angle, the CT stopped or bounced without significant compression. We investigated many aspects of the dynamics of CT ring acceleration, including the limits on the accelerating force, ring shaping in response to acceleration, and the balance of $C T$ equilibrium forces with the accelerating force in the precompressor cone. In those experiments, we achieved good correspondence with two-dimensional (2D) magneto-hydrodynamic (MHD) and other models, especially for the precompression cone experiments where we found excellent agreement between our observations and 2D simulations using the TRAC code $^{10}$.

The configuration of the RACE facility, during the experiments described in this section, is shown in Fig.1.2. For these experiments, we installed a new set of electrodes combining all four phases: formation, precompression, acceleration, and focusing. As in the previous experimental runs, the CT is formed at the muzzle of a magnetized, coaxial plasma gun and is initially captured in the precompression section. The precompression section consists of two coaxial metal cones. The cones have an outer diameter of $0.32 \mathrm{~m}$ (inner diameter of $0.14 \mathrm{~m}$ ) that converges a factor of 2 over an axial distance of $0.43 \mathrm{~m}$. The electrode geometry is self-similar 
throughout; i.e., the ratio of inner to outer electrode diameters is independent of position along the accelerator. If the accelerator bank is not fired, the $\mathrm{C}^{-}$-emains at the large-radius end of the precompressor and persists as a stable-syr etric structure, decaying within about $100 \mu \mathrm{s}$.

When the accelerator bank is fired, the CT is slowly compresse relauve to the Alfven time which is the order of $1 \mu \mathrm{s}$ ) over a period of $10 \mu \mathrm{s}, \mathrm{dl}$ ig which the acceleration force approximately balances the expansive force 0 : Ie CT in the precompressor cone. The expansive force is similar to the force on .. Jalloon that has been inserted into a smooth, conical tube. The balloon, or CT, exerts a pressure against the walls of the tube, which results in a net force toward the side end of the cone. During this time, accelerator field energy is inductively stored behind the CT.

The CT is compressed the factor of two in an axial distance of $0.43 \mathrm{~cm}$, then it enters the acceleration/focusing section. This section is also conical; the outer diameter shrinks another factor of three from 0.15 to $0.053 \mathrm{~m}$ over an axial distance of $7.4 \mathrm{~m}$. The cone angle is much shallower than it is in the precompression section, so the expansive force plays a smaller role. In fact, the cone angle was made as small as possible, by making the length of the cone equal to the length of the accelerator, to preclude the bouncing or non-flux-conserving behavior that had been previously observed. The acceleration force is mainly balanced against the acceleration of the CT and any drag forces. At the smalldiameter end, the CT exits the accelerator and is allowed to expand.

The electrodes are constructed from aluminum to minimize the magnetic skin depth, and faced with refractory metals to reduce sputtering. The inner electrode is coated with plasma sprayed tungsten to a thickness of about $0.05 \mathrm{~cm}$. The outer electrode is covered with $0.025 \mathrm{~cm}$ thick tantalum sheet that has been hydroformed to conform tightly to the aluminum structure. The outer electrode is interrupted at approximately $0.7 \mathrm{~m}$ intervals with rings that provide penetrations for diagnostics. These rings are also coated with plasma sprayed tungsten to a thickness of about $0.05 \mathrm{~cm}$.

The major diagnostic used for the discussion in this section is $\mathrm{t}: \mathrm{a}$ magnetic loop probe system that measures the axial (poloidal) and theta (toroida: components of the magnetic field at 11 axial locations on the inner wali of the outer electrode, after the precompressor. At most locations, 2 pairs of probes are installed with toroidal separations of $180^{\circ}$. Other diagnostics include $\mathrm{HeNe}$ interferometers located at three axial locations, a visible light spectrometer to measure the time dependence of oxygen impurity lines, and X-ray diodes and photo diodes to measure VUV emissions at several axial locations. 
The trajectory of a moving compact torus is displayed by a family of time plots of magnetic field in Fig. 1.3. The vertical spacing of the plots is proportional to the axial spacing of the magnetic probes. The characteristic signature of a moving CT is a localized poloidal field signal as the CT passes over a probe location. The slope of a line fitted to the time of peak field gives its velocity. In this example the CT accelerated to $5.9 \times 10^{7} \mathrm{~cm} / \mathrm{s}$, although we have observed velocities up to $1.5 \times 10^{8} \mathrm{~cm} / \mathrm{s}$ in this configuration of RACE.

The trajectory line shown is from the zero-dimensional (OD) RAC code 2 . For this calculation, we varied the CT mass to give the best fit, resulting in a mass of $62 \mu \mathrm{g}$ and a kinetic energy of $11 \mathrm{~kJ}$ (the accelerator bank energy was $65 \mathrm{~kJ}$ ). From the interferometer measurement of the chord-averaged electron density, we obtain a mass estimate an order of magnitude lower, assuming hydrogen as the dominant constituent. The difference may be due to the carbon and oxygen impurities that we observe spectroscopically. If oxygen is the dominant CT constituent, the interferometer-based estimate would be much closer to the dynamically based estimate. The difference may also be due to anomalous drag from interaction of the plasma with gas evolved from the walls. Additional drag would reduce the mass needed in the RAC code to fit the experimental CT trajectory.

Of particular note for the shot shown in Fig. 1.3 is the increase in magnetic field as the CT is compressed. During acceleration, the $C T$ passes a given point more quickly, both because it is becoming shorter and because its velocity is increasing. Both of these observations will be quantified for comparison with MHD theory in subsequent figures.

To compare the observed magnetic field amplification during compression with that predicted for flux conservation, we plot the poloidal magnetic field vs the reciprocal of the outer electrode radius in Fig. 1.4. We see that the magnetic field is decaying, or constant, for the first $3 \mathrm{~m}$ (the first five data point sets) of axial distance. After that, the magnetic field increases. For comparison a $(1 / R)^{2}$ line is fit to this part of the data. We conclude that conservation of poloidal flux is a good approximation during the final $4 \mathrm{~m}$ of acceleration over which the magnetic field increases by a factor of five. This result is consistent with ideal (zero electrical resistivity) MHD theory.

We determine the length of the CT in two ways. First, we measure the full width (in $\mu s$ ) at half maximum of each of the probe locations shown in Fig. 1.3, and multiply this by the local velocity to get the CT length as it passes that location. The length is found to be approximately proportional to the radius as shown in Fig. 1.5a. A second method is to find a shape and length for the plasma at 
each time by fitting a profile to signals simultaneously present on two to ar probes. The length obtained in this way is plotted vs axia! position in Fig. 5b, along with a line with its length proportional to radius for companson. $T$ atter line is an approximate fit to the data between 5 and $7 \mathrm{~m}$. We find that th $H$ J prediction that length of a CT is proportional to the radius is approxima satisfied.

Good agreement with flux-conserving MHD theory is obtained y during the final $4 \mathrm{~m}$ of acceleration. The initial decay may indicate ohmic decay at electron temperatures of 3-5 eV. The CT magnetic field following the precompression stage and at the beginning of the acceleration/focusing stage is irregular, suggesting that the drive magnetic field at that time was excessive. High drive field causes the "blowby" effect where accelerating field blows past the CT, either partially or completely disrupting the CT. The robustness of the CT geometry is indicated by the coalescence of multiple peaks into a single peak as the CT is accelerated.

\section{Drag and Heated Electrode Studies}

\subsection{Experiments}

\subsubsection{Drag measurements and phenomenology}

Drag is generally associated with the exchange of CT momentum with plasma or gas evolved from the walls either before or during the passage of the CT. Classical resistive drag with the electrodes is small in the RACE operating regime. Some OMA (optical multi-channel analyzer) data from the RACE long-cone accelerator showed the light emission peaking at the front of the CT, rather than at the back where the density peaks. In addition, the emission shows a transition from predominately singly ionized oxygen at early times to triply ionized as the rear end of the CT sweeps past a location. An example, Shot 12054, is shown in Figs. 2.12.3. These data suggest that gas at a significant density exists in the gap between electrodes, and that the CT is ionizing and sweeping up the gas as the $C^{-}$transits the accelerator.

We examine the hypothesis that sufficient gas exists between the e rodes to cause the drag that prevents the compact torus from accelerating. From $2.2 \mathrm{a}$, we see that the density at $194 \mathrm{~cm}$ has a nearly constarii vensity foot $\epsilon$ ending from the front of the $B_{Z}$ magnetic field at $25 \mu \mathrm{s}$ to the density peak at the $t$. ck of the CT, beginning at $31.2 \mu \mathrm{s}$. At the CT velocity of $14.6 \mathrm{~cm} / \mu \mathrm{s}$, Fig. $2.3 \mathrm{a}$, the foot has a line density of $3.5 \times 10^{16} \mathrm{~cm}^{-3}$, more than enough to completely ionize any gas in front of the CT. We conjecture that the density of the foot is composed completely of gas, swept up from in front of the C.T, which would allow us to 
equate the electron density in the foot to the neutral density before the CT. The momentum balance is given by

$$
F=\frac{\Delta p}{\Delta t}
$$

or

$$
\frac{B_{T}^{2} A}{2 \mu_{0}}=\frac{n_{0} m_{i} A(\Delta z) v_{C T}}{\Delta t}
$$

since $(\Delta z / \Delta t)=v_{C T}$, and assuming that the gas fills the gap so that the areas, $A$, cancel, we obtain

$$
n_{0}=\frac{B_{T}^{2}}{2 \mu_{0}} \frac{1}{m_{i} v_{C T}^{2}}
$$

Evaluating $\mathrm{B}_{T}^{2}$ as the change in the toroidal field pressure between the front and back of the CT, and assuming that the gas is water from the walls with a total mass of 18 AMUs and results in 2 singly charged hydrogen and 1 doubly charged oxygen (From Fig. 1, OIII which is doubly charged dominates except at the front of the CT where OII dominates and the back where OIV dominates.) yielding and average ion mass of 4.5 AMUs. With these assumptions, we compute an electron density of $6.5 \times 10^{14} \mathrm{~cm}^{-3}$, which is of the same order of magnitude as the measured electron density of $4.6 \times 10^{14} \mathrm{~cm}^{-3}$ at $194 \mathrm{~cm}$.

We apply this same analysis to shots $12045-12062$. In Fig. 2.4, we plot the measured electron density in the front foot of the CT vs $\mathrm{n}_{0}$ from Eq. 3 at an axial position of $194 \mathrm{~cm}$. In Fig. 2.5, we show the same plot at $120 \mathrm{~cm}$, but using the measured electron density at 194 since we did not have an interferometer at 120 $\mathrm{cm}$. In both figures, a unity slope line is shown for comparison. The computed neutral density required to prevent acceleration of the CT is somewhat greater than the measured electron density in both cases. Closer agreement can be obtained by using an average mass of 6 (for singly charged oxygen) rather than 4.5 AMU, however, the spectroscopic data indicate that 4.5 is the better value.

Two classical processes would also reduce the neutral density required for the sum of all drag processes to create a constant velocity CT. These are the drag due to the magnetic field soaking into the electrodes and the back force on the CT due to the conical electrodes. Both of these effects are predicted to be small.

We now discuss the source of the gas. Vacuum systems generally have the order of 100 monolayers of gas adsorbed on the walls 13 , which can be readily desorbed by plasma ions incident on the wall at energies the order of $100 \mathrm{eV} 14$. Many neutrals per incident ion may be released, depending on the surface 
conditioning. We observe that a prepulse plasma frequently extends $1-2 \mathrm{~m}$ down the accelerator for a period of 10 's of $\mu$ s before the CT traverses the accelerator. This plasma is very apparent on Langmuir probes, and is also seen with mar netic probes, VUV's, VUV monochromators, and to a slight degree with interfer leters.

Langmuir probe data from a probe inserted to $1.0 \mathrm{~cm}$, is shown in Fig. 2. a from Shot 12218 . The Langmuir probe is shielded by at tantalum cap with a pinrisle to admit plasma. The probe was at $z=120 \mathrm{~cm}$, with the pinhole facing towards the gun. Fig. 2.6b shows the accumulation of poloidal magnetic field at the entrance to the precompressor at $z=12 \mathrm{~cm}$. Three spikes of plasma emission are seen in the Langmuir probe current between 1 and $7 \mu \mathrm{s}$, which is near the peak poloidal field buildup at $12 \mathrm{~cm}$. The CT passes the Langmuir probe between 20 and $30 \mu \mathrm{s}$, which gives a much smaller ion current.

Rotating the Langmuir probe to look away from the gun eliminates the spikes, as shown in Fig. 2.7 for Shot 12214, indicating that the prepulse ion flux is directed downstream from the gun. Surprisingly, the CT current at $27-30 \mu$ s is several times larger than the upstream signal of shot 12218 , indicating that the plasma ions near the wall may be flowing in the direction opposite to the CT.

In Fig. 2.8., the probe is inserted another $0.5 \mathrm{~cm}$ to $1.5 \mathrm{~cm}$, and faces the gun, Shot 12221. Here, the prepulse ion current is smaller, the CT ion current is larger. From Figs. 2.6-8, we conclude that fast prepulse ions are due to emission from the plasma gun, and that they are close to the wall. This may be appropriate for ions resulting from charge exchange or ionization of cold gas in front of the CT. The gas is concentrated near the electrodes because in $-20 \mu \mathrm{s}$ it can move only a short distance given by

$$
\delta r(\mathrm{~cm})=v_{0} \delta=10^{6} \mathrm{~cm} / \mathrm{s}\left(\frac{2}{A}\right)^{0.5} E_{0}^{0.5}(\mathrm{eV}) 2 \times 10^{-5} \mathrm{~s}=1.1 \mathrm{~cm}
$$

where we assume that the desorbed neutrals are $\mathrm{H}_{2} \mathrm{O}$ at a temperature of $300 \mathrm{~K}$. The electrode gap was in the range 1.5 to $4.4 \mathrm{~cm}$, so even gas at $300 \mathrm{~K}$ can fill at least half of the gap. Higher energies are possible, since the ions impinge at glancing incidence on the walls, and could impart a significant fraction of t eir energy to the $\mathrm{H}_{2} \mathrm{O}$.

To estimate the ion velocity and energy, we look at the time of flight from other diagnostics.

UV diagnostics are shown in Figs. 2.9-11. Fig. 2.9 shows a UV monochromator signal at $28 \mathrm{~cm}$ (within the precompressor cone that extends from 
$z=16$ to $38 \mathrm{~cm}$ ). Fig. 2.10 shows a VUV (XRD secondary emission detector) at 46 $\mathrm{cm}$, just after the precompressor. Spikes are seen in both detectors, that appear correlated with those in the Langmuir probe current. The large spike in Figs. 2.9 and 2.10 is associated with the passage of the CT. Fig. 2.11 shows another VUV detector at $\mathrm{z}=194 \mathrm{~cm}$, which exhibits very small spikes or noise, between $0 \mu \mathrm{s}$ and the CT arrival at $28 \mu \mathrm{s}$, that may correlate with other diagnostics. Deriving velocities from these data, we obtain $2.1-3.7 \times 10^{6} \mathrm{~cm} / \mathrm{s}$ between 28 and $46 \mathrm{~cm}$, and $2.4-3.4 \times 10^{7} \mathrm{~cm} / \mathrm{s}$ between 46 and $194 \mathrm{~cm}$. . Helium ions moving at $2.4-$ $3.4 \times 10^{7} \mathrm{~cm} / \mathrm{s}$ have an energy of $1.1-2.3 \mathrm{keV}$.

Magnetic probes measuring the poloidal field at $z=43,120$, and $194 \mathrm{~cm}$ are shown in Fig.s 2.12-14 respectively. These signals do not show the strong spikes seen on the previous diagnostics, but do show 5-20\% modulation of the signal that may correlate with the spikes. The initial rise of the signal propagates from 43 to $120 \mathrm{~cm}$ in about $1 \mu \mathrm{s}$, but takes $5.2 \mu \mathrm{s}$ to reach $194 \mathrm{~cm}$, implying a velocity between 120 and $194 \mathrm{~cm}$ of $1.4 \times 10^{7} \mathrm{~cm} / \mathrm{s}$ or a helium energy of $400 \mathrm{eV}$.

The dominant signal from the magnetic probes shows a poloidal magnetic field extending along the accelerator shortly after a CT begins to form in the precompressor, as seen in Figs. 2.12-14 and Fig. 2.15, for Shots 5877 and 5333. The prepulse magnetic field is generally stronger, closer to the precompressor (which extends from $0.12 \mathrm{~m}$ to $0.43 \mathrm{~m}$ ), decaying to smaller values by $\mathrm{z}=1-2 \mathrm{~m}$. One possibility is that the magnetic field carries little plasma, but pushes prepulse ions against the electrodes. Also, doubly-ionized helium ions at $400 \mathrm{eV}$ (if this represents a temperature as well as a directed velocity) would have a gyroradius of order $3 \mathrm{~cm}$ in the $\sim 1 \mathrm{kG}$ pre-pulse magnetic field and a high probability of intercepting an electrode. The presence of the pre-pulse field in the accelerator implies plasma within the field to carry the self-consistent currents, and either needs excess $\mathrm{B}_{\text {theta }}$ or the momentum of fast ions to balloon the $\mathrm{B}_{\text {poloidal field }}$ axially.

\subsubsection{Heated electrode experiments}

We modified RACE to test the importance of volatile gas emission from the electrodes by installing a set of accelerator electrodes which can be operated at high temperature. The modified electrodes are shown schematically in Fig. 2.16.

Data from the experimental run from February - March 1993 indicates that high temperature $\left(-400^{\circ} \mathrm{C}\right)$ bakeout has little effect on $\mathrm{CT}$ dynamics for some experimental conditions, but may significantly increase the CT velocity for other conditions. Impurity line emissions measured with the OMA were reduced. Fig. 2.17 shows the CT velocity calculated from the transit time between two axially- 
offset interferometers vs. shot number. Three different experimental conditions were tested before, during, and after bakeout. There is a large amount of shot-toshot scatter, but the mean velocity for high inductance, 40 and $50 \mathrm{kV}$ acce! ator voltage shots increased by about $30 \%$ for the hot or post-bakeout tests in comparison with the pre-bakeout tests. Low-inductance, $70 \mathrm{kV}$ shots shc $\mathrm{d}$ no measurable change in velocity with bakeout. The $70 \mathrm{kV}$ shots show a $\mathrm{m}$ a greater difference in velocity when compared with earlier, non-bakeable sold plated, perforated aluminum electrodes.

In addition, we extended the high-inductance operation to 70 and $80 \mathrm{kV}$ accelerator voltage ( $80 \mathrm{kV}$ is the present upper linit on bank operation) with the electrodes hot and observed velocities greater than $50 \mathrm{~cm} / \mu \mathrm{s}$. Previous cold operation in this parameter range yielded velocities of order $20 \mathrm{~cm} / \mu \mathrm{s}$. Fig. 2.18 shows the CT trajectory (axial distance vs. time) for a $70 \mathrm{kV}$ high inductance shot as indicated by peak signals on various diagnostics.

These electrodes had a stagnation region at the end of the accelerator. Data from soft $\mathrm{x}$-ray diodes and silicon photodiode bolometers indicates a strong burst ( $\sim \mu$ s duration) when the CT impacts the stagnation region, followed by a lower, decreasing intensity over 10's of $\mu \mathrm{s}$. This may indicate a long lived post-shock heated plasma.

\subsection{Modeling of Instability of the Wall Plasma Layer in a Coaxial Accelerator}

The dense plasma layer generated by desorption from electrode surfaces is also of concem because this material may become distributed across the interelectrode gap and cause current shunting and reduced power flow to the load. The magnetic pressure of the accelerator is presurned to play a role in the dynamics of the wall-derived plasma. As described by J. Degnan "I , during the current ramp up phase magnetic field diffuses into the plasma and pins it against the wall due to magnetic pressure. During the current decay phase the flux in the wall plasma is at least partly "frozen in" and the plasma expands into the gap as the magnetic pressure drops.

If the current rises and falls slowly enough, we expect the wall plasma to be in near magnetic pressure balance with the field in the gap, where we assume a much lower density plasma exists. Under these conditions, and for sub-Alfvenic flow in the low density region, there is the possibility of instability due to the Venturi effect. If the surface of the dense wall plasma develops a ripple, then the flow of low density plasma becomes locally constricted. In the constriction the pressure drops ( for sub-Alfvenic flow) which causes the wall plasma to expand and enhance the ripple. In the low Alfven Mach number (incompressible) limit 
this goes over to the Kelvin-Helmholtz instability. For Mach numbers close to unity, the instability grows rapidly.

A simple ideal MHD analysis of a high aspect ratio coaxial accelerator is given in the following. Estimates for the most unstable modes (shortest wavelength modes consistent with approximations, i.e., wavelength $\sim$ few $x$ gap ) give growth times in the few - $10 \mu \mathrm{s}$ range, depending on wall plasma density, thickness, etc. The instability may place more severe limits on wall plasma evolution or acceleration time than the expansion during current decay described above.

The interelectrode region (see Fig. 2.19) is divided into a dense wall layer with density, $\rho_{1}$, axial velocity, $v_{1}$, and thickness $\delta_{1}$, and a low density channel with corresponding quantities $\rho_{2}, v_{2}, \delta_{2}$. A uniform (in the high aspect ratio limit) azimuthal field fills the gap and the plasma pressure is assumed to be negligble. A sharp boundary is assumed to exist between regions and the electrodes and plasma are taken to be perfect conductors In this case we can write the nozzle flow equations for either region 1 or 2 :

$$
\begin{aligned}
& \partial(\rho \delta) / \partial t+\partial(\rho \delta v) / \partial z=0 \\
& \partial(\rho v \delta) / \partial t+\partial\left(\rho \delta v^{2}\right) / \partial z=-\delta \partial\left(B^{2} / 2\right) / \partial z \\
& (\partial / \partial t+v \partial / \partial z) B / \rho=0 \\
& B_{1}=B_{2} \\
& \delta_{1}+\delta_{2}=\text { constant }=\text { interelectrode gap }
\end{aligned}
$$

We look for perturbations around a steady flow state, uniform in $z, t$. The unperturbed flow velocity near the wall, $v_{1}$, is taken to be 0 . For region 2 we assume that the perturbations have a frequency $\omega<<k v_{A_{2}}, k v_{2}$, where $v_{A 2}=B / \rho_{2}^{1 / 2}$ and $k$ is the axial wavenumber. In both region 1 and 2 the perturbations are proportional to $\exp (-i \omega t+i k z)$. In the low frequency case we can neglect the time derivatives in equations 2.2.1-3. Equations 2.2.1 and 2.2.3 can then be integrated directly with the results substituted into 2.2 .2 , giving the familiar nozzle equation:

$$
\left(v_{2}^{2}-v_{A 2}^{2}\right) \partial \rho_{2} / \partial z+\rho_{2} v_{2}{ }^{2} / \delta_{2} \partial \delta_{2} / \partial z=0
$$

For small perturbations, $\rho_{2}=\rho_{20}+\Delta \rho_{2}, \delta_{2}=\delta_{20}-\xi$, we have

$$
\Delta \rho_{2} / \rho_{20}=\Delta B / B=\left(v_{2}^{2} /\left(v_{2}^{2}-v_{A 2}^{2}\right)\right) \xi / \delta_{20}
$$


For region 1 the linearized equations, with $\delta_{1}=\delta_{10}+\xi$, are:

$$
\begin{aligned}
& \Delta \dot{p}_{1} \delta_{10}+\dot{\xi} \rho_{10}+\rho_{10} \delta_{10} \partial v_{1} / \partial z=0 \\
& \rho_{10} \delta_{10} \dot{v}_{1}=-\delta_{10} B \partial \Delta B / \partial z \\
& \Delta B / B=\Delta p_{1} / \rho_{10}
\end{aligned}
$$

The region 1 equations reduce to

$$
\left(\omega^{2}-k^{2} B^{2} / \rho_{10}\right) \Delta B+\omega^{2} B \xi / \delta_{10}=0
$$

Equation 2.2.11 can be combined with equation 2.2 .7 to give a dispersion relation:

$$
\omega^{2}=-k^{2} v_{2}{ }^{2}\left(\delta_{10} / \delta_{20}\right)\left(\rho_{20} d \rho_{10}\right) /\left(1-v_{2}{ }^{2} / v_{A 2}{ }^{2}\left(1+\delta_{1 d} \delta_{20}\right)\right) \text {. }
$$

This expression indicates instability for all sub-Alfvenic flows with

$$
v_{2} / v_{A 2}=M<M_{\text {crit }}=1 /\left(1+\delta_{1 d} \delta_{20}\right)^{1 / 2}
$$

The growth rate diverges as $M->M_{\text {crit }}$, however the assumption $\omega<<v_{2}$ breaks down at this point. For Mach numbers, $M$, not close to $M_{\text {civi }}$ the assumption will usually be satisfied since $\delta_{10} d \delta_{20}, \rho_{20} d \rho_{10}$ are $\ll 1$ for cases of interest. The fastest growing modes are at the shortest wavelength. The growth rate is unbounded as $k$ becomes large, a consequence of the nozzle flow, sharp boundary approximations. In practice, the largest $\mathrm{k}$ consistent with the approximations is of order the (interelectrode gap) $)^{-1}$. For the $\mathrm{CT}$ accelerator the flow in the low density region is typically sub-Alfvenic (if not, most of the accelerator energy will go into accelerating the plasma in the channel rather than the CT) so the instability is usually present. For example if we assume $M \ll 1$ and take $v_{2}=10^{8} \mathrm{~cm} / \mathrm{sec}, \mathrm{k}=.1$ $\mathrm{cm}^{-1}, \rho_{1}=10^{3} \rho_{\delta}$, and $\delta_{1}=.1 \delta_{2}$, we obtain $\gamma=10^{5} \mathrm{sec}^{-1}$. Growth times are then of order typical acceleration times.

\section{MARAUDER Experiments and Modeling}

The LLNL CT acceleration program has included a collaboration with the Air Force Phillips Laboratory in Albuquerque, New Mexico on the MARAUDER experiment. One configuration of the MARAUDER formation/acceleration electrodes are shown schematically in Fig. 3.1. The goal of these experiments is to demonstrate $C T$ acceleration at the megajoule energy scale and test the CT $x$-ray source concept. We have predicted that stagnation of plasmas with intermediate to high atomic numbers against a barrier should lead to strong shock heating of the 
$C T$ and an intense source of multi-keV $x$-rays, appropriate for a weapons effects simulator.

Our efforts in the collaboration have concentrated on deployment of diagnostics and modeling of CT acceleration, compression and stagnation . We deployed a 16 channel detector to measure the space and time-resolved vacuum UV emission during acceleration, giving an indication of how the plasma is distributed. The detector, shown in Fig. 3.2, views different radial chords at a fixed axial location. The data are Abel inverted, assuming axial symmetry, to give the radial dependence. Fig. 3.3 shows the emitted power as a function of radius and time for a MARAUDER shot. This shot, employing neon gas in the CT formation, produced significant $\mathrm{keV} x$-ray yield at stagnation. The data are collected about halfway along the acceleration/focusing cones, between the formation and stagnation regions. Most of the emission comes after the passage of the CT and is localized near the electrodes, as expected for plasma produced from electrodedesorbed gases. Spectroscopic observations suggest that the wall plasma is largely composed of carbon and oxygen, which are common contaminants. The localization near the electrodes is consistent with current and voltage measurements that show the accelerating current is flowing at the moving CT rather than being "shorted out" in the following plasma.

We also deployed a data acquisition system similar to the RACE system, Langmuir probes to sample the edge plasma density, visible light framing and streak cameras to view the stagnation region, and new magnetic probes, flush mounted in the electrode surface, to allow measurements toward the muzzle of the accelerator. The probes allow us to obtain better data on the CT trajectory and field structure without the probe ablation and perturbative effects of the existing insertable probes.

We have used the two dimensional TRAC2 magnetohydrodynamics code to model CT dynamics in MARAUDER. We have used the code to evaluate different designs for the transition between conical and straight electrodes. The code predicts that a geometry with a gradual transition, the "gentle corner" design ,should greatly reduce the non-adiabatic heating in comparison with earlier abrupt transitions. Excessive plasma heating at a transition can have severe effects on the acceleration process, such as disruption of the CT or ablation of wall material from the resulting intense $x$-ray pulse.

The sharp/gentle electrode geometries are sketched in Fig. 3.4. For all simulations, a CT in the Taylor state is initialized in the region just upstream of the comer with varying velocities. The CT parameters are mass $=.9 \mathrm{mg}$, length $=20$ $\mathrm{cm}$, magnetic energy $=3.5 \mathrm{~kJ}$, thermal energy $=1 \mathrm{~kJ}$, peak magnetic field $=10 \mathrm{kG}$, electron temperature $=$ ion temperature $=30 \mathrm{eV}$, atomic mass $=20, Z_{\text {eff }}=4$. 
The calculations, summarized in table 3.1 telow, do not include selfconsistent ionization balance, ohmic losses or radiation. The non-adiabatic $\Delta U_{\mathrm{NA}}$, is the sum of the artificial viscosity and energy conservation terms. ich are of comparable magnitude. Shock heating is manifested in the artificic viscosity terms in MHD numerical calculations. The increase in therma: $\Delta \mathrm{U}_{\mathrm{Tl}}$, is the sum of non-adiabatic and $\mathrm{PdV}$ terms. The change in energ: :aken at a time after the $C T$ rounds the comer when the kinetic energy is minim. (subsequent expansion of the CT can convert some of the thermal ener. $t_{\text {. }}$ into kinetic energy).

Table 3.1

$v(\mathrm{~cm} / \mu \mathrm{s})$

20.

30.

40.

50.

$$
\underset{\Delta U_{T H} / U_{K N}}{\text { Sharp }}
$$

.046

.040

.040

.035
Gentle $\Delta \mathrm{U}_{\mathrm{TI}} / \mathrm{U}_{\mathrm{KN}}$

.020

.010

.006

.005

$$
\begin{array}{cc}
\text { Sharp } & \text { Gentle } \\
\Delta U_{\mathrm{NA}} / \mathrm{U}_{\mathrm{KIN}} & \Delta \mathrm{U}_{\mathrm{NA}} / \mathrm{U}_{\mathrm{KIN}}
\end{array}
$$

.021

.0014

.0012

.0013

.0020

The sharp comer calculation shows heating roughly as expected if the radial part of the CT kinetic energy is converted to heat, i.e., $\Delta \mathrm{U}_{T H} / \mathrm{U}_{\mathrm{KN}}=\left(\mathrm{v}_{\mathrm{RADIA}} / \mathrm{v}\right)^{2}=$ .05. This type of "inelastic collision" with the electrodes while preserving the axial momentum was seen in earlier TRAC2 runs.

We have aiso used LASNEX to model $x$-ray output at stagnation. These calculations are ID with the CT compression ratio (initial/final radius), length, density, magnetic field, and velocity taken from slug model predictions of CT parameters at the muzzle of the accelerator. Calculations of $x$-ray output in various spectral energy ranges are shown in fig. 3.5 for MARAUDER and a hypothetical $50 \mathrm{MJ}$ facility. Only the lowest velocity example thas actually been achieved on MARAUDER. Longer acclerator geometries than have been currently tested would be required to reach high velocity ( $>40 \mathrm{~cm} / \mu \mathrm{s}$ ). Predicted spectra for sor:: $:$ of the parameters in Fig. 3.5 are shown in Fig. 3.6

\section{Accelerator Variants}

\subsection{Radial CT Accelerator}

It may be possible to reach high velocities $(-100 \mathrm{~cm} / \mu \mathrm{s})$ and high power densities $\left(10-100 \mathrm{TW} / \mathrm{cm}^{2}\right)$ without using a long acceleration stage, but instead a pure radial compression geometry. This could be an advantage for reducing wall 
effects since the electrode area is small. The concept is based on inductive storage behind the CT in non-self-similar electrode geometry and radiation clamping of the CT temperature.

We have usually considered self-similar geometry ( $g$ =interelelectrode gap / mean radius =constant) for the CT accelerator although MARAUDER has employed non-self-similar electrodes in one series of experiments ${ }^{9}$. There may be an advantage to some types of non-self-similarity, e.g., the inductance of the accelerator can increase rapidly with distance if $\mathrm{g}$ increases, allowing efficient transfer of inductively stored energy to CT kinetic energy in a short accelerator. Another aspect of non-self-similarity is the different variation of the CT magnetic energy with radius. In the approximations usually employed in OD models such as the RAC code ${ }^{2}$, ( CT length $\ll$ electrode length, $\mathrm{g} \ll 1, \beta \ll 1$ ) the CT magnetic energy varies as (electrode gap) ${ }^{-1}$ and hence inversely with radius for selfsimilarity. If the radius decreases but the gap does not change, however, there is no change in the CT magnetic energy and no corresponding restoring force, to first order in the approximations mentioned above.

A purely radial geometry is interesting for exploiting non-self-similarity since it is less prone to blow-by as $\mathrm{g}$ increases (there is no difference in magnetic pressure between inner and outer electrodes). Fig. 4.1b shows one example where conically converging electrodes transition to electrodes with constant gap. The behavior of the accelerator, based on OD model calculations, is similar to other MARAUDER geometries. The circuit used in these calculations, representing the SHIVA-star bank, is shown in Fig 4.1a. The calculations show a period of 5-10 $\mu \mathrm{s}$ of current build-up with the piston force nearly balancing the equilibrium force of the CT, followed by a rapid increase in inductance and transfer of inductive energy to kinetic energy. We have done a OD calculation for full energy MARAUDER $(9.4 \mathrm{MJ})$ with the circuit and electrode geometries of Fig. $4.1 \mathrm{a}, \mathrm{b}$. Since the voltage climbs to $3 \mathrm{MV}$ as the CT implodes, most of the inductance should be on the vacuum side of the insulator. The model neglects drag and finite beta and assumes a magnetic decay time proportional to the square of the gap distance. The decay model is consistent with constant, radiatively clamped electron temperature. The CT parameters were: mass $=1 . e-3$ grams, magnetic energy $=100 \mathrm{~kJ}$, length $=15 \mathrm{~cm}$ and initial magnetic energy decay time of $20 \mu \mathrm{s}$.

The CT reaches a kinetic energy of $660 \mathrm{~kJ}$ and a radial velocity of $115 \mathrm{~cm} /$ $\mu$ s at $C T$ mean radius, $R_{C T}=.2 \mathrm{~cm}$, where the calculation is arbitrarily stopped. The efficiency is only $7 \%$. It is hard to do better than this with the series resistance shown in Fig 4.1a. Lowering the series resistance, reducing external inductance and optimizing the geometry should improve efficiency substantially. The CT geometry is strongly distorted at a radius of $.2 \mathrm{~cm}$ and the OD model approximations are no longer valid. More accurate calculations will require 2D 
MHD simulations. Essentially, the CT implosion transitions to a (possibly stabilized) z-pinch implosion.

If the CT is too small in radial extent, magnetic tension forces car $C T$ away from the electrodes. The CT length (CT radial extent in this ge varies approximately proportional to $\mathrm{R}_{\mathrm{CT}}$, so at sinall enough radius, the equals the gap distance. If the length becomes shorter than the gap dis CT will also contract axially, possibly causing detachment of the curr blowby. For our example, the length would equal the gap distance at The time to pull away from the electrodes is roughly the gap distance, er the Alfven velocity $\sim 2 \mathrm{~cm} / 40 \mathrm{~cm} / \mu \mathrm{s} \sim .05 \mu \mathrm{s}$. This is comparable to the remaining time to implode $\sim 6.7 \mathrm{~cm} / 115 \mathrm{~cm} / \mu \mathrm{s} \sim .06 \mu \mathrm{s}$, so detachment may be an issue in the final phase. The tendency toward detachment could be countered by introducing axial magnetic flux crossing the gap that reconnects with the CT flux as it implodes. If all of the CT flux were reconnected in this fashion, the final configuration would resemble a $B_{z}$-stabilized z-pinch ${ }^{12}$. Alternatively, a CT with sufficiently large initial length could be used to prevent detachment. The final stagnation phase of the CT on axis should be dynamically similar to z-pinch implosions where the plasma kinetic energy is converted to thermal energy and radiation.

\subsection{Low voltage/long drive pulse CT accelerator}

Part of the appeal of the CT accelerator concept has been the comparatively low voltage power sources (few hundred $\mathrm{kV}$ ) that can be used by exploiting inductive storage and precompression. We usually assume that the limitation on the inductive storage time is the CT lifetime. In this section we consider the possibility of using a sustained CT during the current build-up phase to decouple the inductive storage time from the CT lifetime, thereby allowing very low voltage drivers $(\sim 10 \mathrm{kV})$.

The scheme works as follows: a CT can be formed and sustained at the beginning of the precompression cone for long periods, based on the sli.' ainment experiments at LANL ${ }^{12}$. Pump-out holes/slots would be necessary to at 1 buildup of plasma or neutrals (see Fig. 4.2). The accellerator current is then $i$.... oed up over an extended time, e.g., $1 \mathrm{msec}$, with the minimum drive voltage set the resistive loss of the CT, typically $-10^{-5}$ ohms based on plasma resistivity $10 \mathrm{eV}$ and a CT radius of $50 \mathrm{~cm}$. The gun current and helicity injection rate alsc $\mathrm{mp}$ up to maintain sufficient magnetic energy in the CT to keep it from compress g. Fig 4.3 shows typical waveforms for the accelerator current and CT flux ( proportional to the gun current). Near peak current the gun is tumed off and the CT begins to decay. Slightly later the CT starts to compress since its equilibrium force is no longer sufficient to resist the accelerating force at the precompressor breech. 
The CT continues to decay and compress until it "rounds the comer" and takes off down the accelerator tube. This type of behavior was observed on many RACE shots in the precompressor geometry where the initial rise of the accelerator current was insufficient to compress the CT ( at high initial CT field or low accelerator voltage) and the CT was found to compress slowly during its decay with the accelerator current crowbarred.

The power losses for this scheme are dominated by the gun, although an "anomalous" accelerator loss comparable to the gun loss might be expected since the accelerator bank coupling to the CT is similar to the gun (see Fig. 4.2). We can estimate the gun voltage and power from $\mathrm{C}$. Bames empirical gun impedance formula ${ }^{15}$,

$$
\mathrm{V}_{\mathrm{G}}=\mathrm{r}_{\mathrm{G}}\left(\mathrm{I}_{\mathrm{G}}-\mathrm{I}_{\mathrm{C}}\right), \quad \mathrm{r}_{\mathrm{G}} \sim .5 / \mathrm{R}
$$

where $V_{G}$ is in volts, $I_{G}$ is the gun current in amperes and $R$ is the gun radius in $\mathrm{cm}$. $\mathrm{I}_{C}$ is the critical current for overcoming field line tension within the gun,

$$
I_{c}=\lambda_{G} / \mu_{0} \psi_{G} .
$$

$\Psi_{G}$ is the gun poloidal flux and $\lambda_{G}$ is the Taylor eigenvalue for the gun, $\lambda_{G} \sim \pi / \Delta_{G}$, where $\Delta_{\mathrm{G}}$ is the gun interelectrode gap. If steady-state balance between gun injection and CT magnetic decay exists, then we can use helicity injection concepts $^{16,17,11}$ to find the CT magnetic energy. Defining the CT helicity as $\mathrm{K}$ we have

$$
\text { Helicity input rate }=2 V_{G} \Psi_{G}=\text { Helicity decay rate }=K / \tau_{\text {Decay }}
$$

The CT magnetic energy is related to the helicity through the Taylor eigenvalue for the formation region, $\lambda-\pi / \Delta$, with $\Delta$ the formation region interelectrode gap. Making use of equations 4.21-3, we have the CT magnetic energy given by

$$
\mathrm{U}_{\mathrm{M}}=\lambda /\left(2 \mu_{0}\right) \mathrm{K}=\lambda / \lambda_{G} \tau_{\text {Decay }} \mathrm{r}_{\mathrm{G}} \mathrm{I}_{\mathrm{C}}\left(\mathrm{I}_{\mathrm{G}}-\mathrm{I}_{\mathrm{C}}\right)
$$

The magnetic energy is optimized by operating the gun with poloidal flux such that $I_{C}=I_{G} / 2$. At optimum the magnetic energy is then

$$
\mathrm{U}_{\mathrm{M}}=\lambda \lambda_{\sigma} \tau_{\text {Deay }} \mathrm{r}_{\mathrm{G}} \mathrm{I}_{\mathrm{G}}{ }^{2} / 4=\lambda \lambda_{\mathrm{G}} \tau_{\text {Deay }} \mathrm{V}_{\mathrm{G}} \mathrm{I}_{\mathrm{G}} / 2
$$

For this scheme the CT magnetic energy must be sufficient to "plug" up the accelerating field during current rise. Approximate force balance is given by

$$
\mathrm{U}_{\mathrm{M}} / \mathrm{I}_{\text {cone }}=\mathrm{L}^{\prime} \mathrm{I}_{\text {drive }}{ }^{2} / 2, \mathrm{~L}^{\prime}=2 \times 10^{-9} \ln \left(\mathrm{R}_{\text {outer }} / \mathrm{R}_{\text {irmer }}\right)
$$


with $l_{\text {cone }}$ the length of the precompressor cone, $L$ ' the inductance per unit ! : gth of the accelerator, and $\mathrm{I}_{\text {drive }}$ the accelerator driving current. Equations 4.2.5,f low us to relate the gun power to the accelerator drive current.

$$
\mathrm{P}_{\mathrm{G}}=\mathrm{V}_{\mathrm{G}} \mathrm{I}_{\mathrm{G}}=\mathrm{Z}_{\text {lost }} \mathrm{I}_{\text {drive }}{ }^{2}, \quad \mathrm{Z}_{\text {loss }}=\left(\lambda_{\mathrm{G}} / \lambda\right) \mathrm{L}^{\prime} \mathrm{l}_{\text {cone }} / \tau_{\text {Decay }} \quad(. \quad . j)
$$

where $Z_{\text {lost }}$ is an effective impedance. For example, if $\lambda_{G} / \lambda=2, L^{\prime}=1(\quad$ one $=100$ $\mathrm{cm}$ and $\tau_{\text {Decay }}=200 \mu \mathrm{s}$ then $Z_{\text {loss }}=10^{3}$ ohms. At accelerator drive volta $z_{i}, \mathrm{~s}$ below $Z_{\text {loss }} I_{\text {drive }}$ the energy losses in the gun will exceed the inductively stored energy, e.g., if the accelerator drive current is $10^{7}$ amperes with $Z_{\text {lost }}=10^{-3}$ ohms, the accelerator drive voltage should exceed $10 \mathrm{kV}$.

We can also relate the gun current to the accelerator drive current through equations 4.2.1 and 4.2.7, since $\mathrm{P}_{\mathrm{G}}=\mathrm{r}_{\mathrm{G}} \mathrm{I}_{\mathrm{G}}{ }^{2} / 2=\mathrm{Z}_{\text {loss }} \mathrm{I}_{\text {drive }}{ }^{2}$.

$$
I_{G}=\left(2 Z_{\text {loas }} / r_{G}\right)^{1 / 2} I_{\text {drive }} \text {, }
$$

For example, if the gun radius is $50 \mathrm{~cm}$, then $\mathrm{r}_{\mathrm{G}}-10^{-2}$ ohms from equation 4.2 .1 and if $\mathrm{Z}_{\text {loss }}=10^{3}$ then $\mathrm{I}_{\mathrm{G}}-.45 \mathrm{I}_{\text {dnive }}$.

One concern with this scheme is the prepulse plasma described in section 2 above. Millisecond drive pulses will allow plenty of time for prepulse-driven desorbed gasses to cross the interelectrode gap. On the positive side, the long pulse may give adequate time for pumpout of the gas.

\section{Conclusions}

Neglecting wall effects, one can use the point model or 2D MHD codes to find a wide variety of possible accelerator geometries that lead to a tightly focused, high velocity, high energy density CT at the accelerator output. Our experience with RACE and MARAUDER, however, suggests, that wall effects place severe constraints on accelerator operating regimes. Unfortunately, determining the source and distribution of wall-evolved material is not very amenable tc 'first principles" modeling since it is dependent on conditioning of surfaces a the interaction of low density pre-pulse plasma with the surfaces. Once the c ribution of material is known, the effect on the CT can be calculated as discussed : section 2 above. While a fundamental understanding of the wall effects remains $e$ ive, some empirical "rules of thumb" can be drawn from the experiments, whic we list here:

The inter-electrode gap during acceleration should be sufficiently large. Sufficiently large means of order $10 \mathrm{~cm}$ for formation/acceleration times of order $10 \mu \mathrm{s}$. Desorbed gas moves at a velocity of order $10^{5} \mathrm{~cm} / \mathrm{s}$, so a gas/plasma layer 
of order a $\mathrm{cm}$ in thickness can form in $10 \mu \mathrm{s}$. The gap should be large compared to this distance. The high velocity CT's observed in earlier RACE and MARAUDER experiments had an electrode gap of $15 \mathrm{~cm}$. Focusing to small size should take place after the CT is at high velocity $\left(-10^{8} \mathrm{~cm} / \mathrm{sec}\right)$ as well as down stream from any prepulse plasma associated with formation. At high velocity, material desorbed during the time of CT passage (eventually $<1 \mu \mathrm{s}$ ) should remain close to the surface.

Fast $(<10 \mu s)$ is better than slow $(\sim 100 \mu s)$ formation . Our last series of experiments on RACE employed slow formation, which produce symmetric, quiescent CT's, but also sent low density $\left(<10^{13} \mathrm{~cm}^{-3}\right)$ plasma streaming down the accelerator tube. We believe this plasma is responsible for desorbing gas that essentially filled the accelerator tube, as discussed in sec. 2 above. The exception may be formation on very long time scales $(>1 \mathrm{~ms}$ ) where plasma streaming down the accelerator may pump out the desorbed gas, as in discharge cleaning techniques.

Discharge cleaning and electrode heating helps. There was ample evidence for conditioning of the accelerator in RACE experiments. Subsequent shots at identical conditions would often show a gradual improvement in CT velocity and trailing plasma - sometimes followed by a gradual worsening. RF glow discharge aided recovery after an air cycle, but did not clearly improve the CT dynamics after the initial conditioning phase. Heating the electrodes to $400^{\circ} \mathrm{C}$ in order to drive off weakly bound gases gave some indications of improvement on RACE, and was found to have very beneficial effects in the CT acceleration experiments at the California Institute of Technology ${ }^{18}$. The RACE heated electrodes, as well as perforated, gold-plated electrodes that were tested, were observed to reduce the "snow plow" through gas seen with the OMA.

\section{Acknowledgements}

We thank Aaron Jones, Richard Green, and Dennis Wilson for operation of the RACE. Richard Cross, Robert Rego, and Jeffery Van Lue with John Delapena, developed hydroforming techniques for expanding tantalum tubes to fit tightly against the aluminum outer electrode. Donald Bubp, Dave Cummings and Dewey Ravenscroft provided capable design and engineering support. Dave Cummings and Marco DiCapua ably assisted with program administration and technical discussions. We gratefully acknowledge the support of J. H. Nuckolls, R. J. Former, and J. F. Holzrichter. This work was performed under the auspices of the 
U.S. Department of Energy at Lawrence Livermore National Laboratory under Contract No. W-7405-Eng-48.

\section{References}

1. C. W. Hartman and J.H. Hammer, Phys. Rev. Lett. 48, 929 (198?..

2. C. W. Hartman and J.H. Hammer, "Acceleration of a Compact orus Jlasma Ring, A Proposed Experimental Study," Lawrence Livermore Nauonal Laboratory Report No. LLL-PROP-191, 15 April 1984. See AIP Document No. PAPS PFBPE03-2236-180 for 180 pages of the RACE experimental Proposal.

3. J. H. Hammer, C.W. Hartman, J.L. Eddleman and H.S. McLean, Phys. Rev. Lett. 61, 2843 (1988).

4. A. W. Molvik, J. L. Eddleman, J. H. Hammer, C. W. Hartman, and H. S. McLean, Phys. Rev. Lett. 66, 165 (1991).

5. J. H. Hammer, J. L. Eddleman, C. W. Hartman, H. S. McLean and A. W. Molvik, Phys. Fluids B 3, 2236 (1991).

6. C. W. Hartman, W. L. Barr, J. L. Eddleman, M. Gee, J. H. Hammer, S. K. Ho, B. G. Logan, D. J. Meeker, A. A. Mirin, W. M. Nevins, L. J. Perkins, D. E. Shumaker, A. W. Leonard, P. B. Parks, H. McLean, E. Morse and D. R. Solvin, in Plasma Physics and Controlled Nuclear Fusion Research, 1988 (IAEA, Vienna, 1989), Vol. 3, p. 547. also,

C. W. Hartman, J. L. Eddleman, J. H. Hammer, B. G. Logan, H. S. McLean, and A. W. Molvik, Fusion Technol 20, 776 (1991).

7. M. Gee, J. H. Hammer, P. F. Nowak, C. W. Hartman, J. L. Eddleman, G. B. Zimmerman and J. F. Holzrichter, Bull. Am. Phys. Soc. 33, 1925 (1988).

8. L. J. Perkins, S. K. Ho, and J. H. Hammer, Nucl. Fusion 28,1365 (1988).

9. J. H. Degnan, R. E. Peterkin, Jr., G. P. Baca, J. D. Beason, D. E. Bell, M. E. Dearborn, D. Dietz, M. R. Douglas, S. E. Englert, T. J. Englert, K. E. Hackett, J. H. Holmes, T. W. Hussey, G. F. Kiuttu, F. M. Lehr, G. J. Marklin, B. W. Mullins, D. W. Price, N. F. Roderick, E. L. Ruden, C. R. Sovinec, P. J. Turchi, G. Bird, S. K. Coffey, S. W. Seiler, Y. G. Chen, D. Gale, J. D. Graham, M. Scott, and W. Sommars, Phys. Fluids B, 5, 2938 (1993).

10. J. L. Eddleman, J.H. Hammer, C. W. Hartman, A. W. Molvik, and H. S. McLean, Bull. Am. Phys. Soc. 34, 2051 (1989). 
11. T. R. Jarboe, I. Henins, A. R. Sherwood, C. W. Barnes, and H. W. Hoida, Phys. Rev. Lett, 51, 39, (1983).

12. R. B. Baksht, A. V. Luchinsky, and A. V. Fedyunin, Zh. Tekh. Fiz., 62, 165 (1992).

13. H. F. Dylla, J. of Nucl. Materials 93 \& 94, 61 (1980).

14. R. A. Langley, et al., "Data Compendium for Plasma-Surface Interactions," Nuclear Fusion Special Issue, 1984.

15. C. W. Barnes, T. R. Jarboe, G. J. Marklin, S. O. Knox, and I. Henins, Phys. Fluids B, 2, 1871 (1990).

16 J. B. Taylor, Phys. Rev. Lett. 33, 1139 (1974), and J. B. Taylor, Rev. Mod. Phys., 58, 741 (1986).

17. W. C. Tumer, G. C. Goldenbaum, E. H. A. Granneman, J. H. Hammer, C. W. Hartman, D. S. Prono, and J. Taska, Phys. Fluids, 26, 1965 (1983).

18. M. R. Brown and P. M. Bellan, Nucl. Fusion, 32, 1125 (1992). and M. R. Brown, P. K. Loewenhardt, J. Yee, D.R. Derkits, and P. M. Bellan, Bull. Am. Phys. Soc., 39, 1596 (1994).

\section{Figure Captions}

Fig. 1.1 A compact torus is a magnetically confined toroidal plasma in near forcefree equilibrium $(\mathrm{j} / / \mathrm{B})$.

Fig. 1.2 The RACE apparatus consists of a magnetized plasma gun, a precompression cone, and an acceleration/focusing stage.

Fig. 1.3 The axial magnetic field at the outer electrode vs time is shown at various axial locations. Each trace is offset vertically by an distance proportional to the axial location. The solid line shows the trajectory predicted by the RAC code, assuming a CT mass of $62 \mu \mathrm{g}$. The axial magnetic field peaks at $8.7 \mathrm{kG}$.

Fig. 1.4 The peak axial field of the CT vs $1 / R$, where $R$ is the $C T$ radius. The line shows the predicted scaling $1 / R^{2}$ for comparison. 
Fig. 1.5 (a) The $C T$ length at each time from fitting the signals on up to 4 magnetic probes. The line shows the predicted scaling $L \propto R$ for comparison. (b) ' length at each location from the full-width (in time) -at-haif-maximum fi. i vs $1 / R$. The line shows the predicted scaling $L \propto R$ for comparison.

Fig. 2.1 (a) Time-dependent optical multichannel analyzer signals for $C$. (dashed curve), OIII (solid and dotted curves) and OIV (dot-dashed curve) em! ion for shot 12054 . OII is observed earliest, followed by OIII and OIV. The sf ctrometer views a radial chord at $z=120 \mathrm{~cm}$. (b) Time-dependent axial magnetic field $\left(B_{z}\right)$ at $\mathrm{z}=120 \mathrm{~cm}$ and for eight azimuthal positions for shot 12054 . The primary CT field shows good symmetry, but the low-field front has a strong $n=1$ asymmetry. The OIV emission peak approximately coincides with the peak of the CT magnetic field.

Fig. 2.2(a) Time-dependent, chord-averaged electron density and axial magnetic field $\left(B_{z}\right)$ at $z=194 \mathrm{~cm}$ for shot 12054 . (b) Same at $z=264 \mathrm{~cm}$.

Fig. 2.3(a) The axial magnetic field at the outer electrode vs time is shown at various axial locations for shot 12054 . Each trace is offset vertically by an distance proportional to the axial location. The straight line through the times of peak axial field gives a CT velocity of $14.55 \mathrm{~cm} / \mu \mathrm{s}$. (b) Axial (dotted curve) and azimuthal (solid curve) magnetic field vs. time at $\mathrm{z}=12,43,120,194$ and $264 \mathrm{~cm}$ for shot 12054.

Fig. 2.4 The vertical axis is the chord-averaged electron density at the front of the CT measured with the interferometer. The horizontal axis is the neutral density inferred from momentum balance. The straight line has unity slope. Quantities are measured at $\mathrm{z}=194 \mathrm{~cm}$.

Fig. 2.5 The vertical axis is the chord-averaged electron density at the front of the CT measured with the interferometer. The horizontal axis is the neutral density inferred from momentum balance. The straight line has unity slope. Quantities are measured at $\mathrm{z}=120 \mathrm{~cm}$, except the interferometer which is at $\mathrm{z}=194 \mathrm{~cm}$.

Fig. 2.6 (a) Time-dependent Langmuir probe current for shot 12218. The probe is inserted $1 \mathrm{~cm}$ from the outer electrode at $\mathrm{z}=120 \mathrm{~cm}$ and faces the gun. (b) Timedependent poloidal magnetic field in the precompressor, $z=12 \mathrm{~cm}$, for shot 12218 .

Fig. 2.7 Time-dependent Langmuir probe current for shot 12214. The probe is inserted $1 \mathrm{~cm}$ from the outer electrode at $z=120 \mathrm{~cm}$ and faces away from the gun. 
Fig. 2.8 Time-dependent Langmuir probe current for shot 12221. The probe is inserted approximately $2 \mathrm{~cm}$ from the outer electrode at $z=120 \mathrm{~cm}$ and faces the gun.

Fig. 2.9 Time-dependent VUV monochrometer signal at $z=28 \mathrm{~cm}$ for shot 12218 . The (negative going) signal shows emission at 1639 angstroms.

Fig. 2.10 Time-dependent $x$-ray diode signal at $z=43 \mathrm{~cm}$ for shot 12218 .

Fig. 2.11 Time-dependent $x$-ray diode signal at $z=194 \mathrm{~cm}$ for shot 12218 .

Fig. 2.12 Time-dependent axial magnetic field signal at $z=43 \mathrm{~cm}$ for shot 12218 .

Fig. 2.13 Time-dependent axial magnetic field signal at $z=120 \mathrm{~cm}$ for shot 12218 .

Fig. 2.14 Time-dependent axial magnetic field signal at $\mathrm{z}=194 \mathrm{~cm}$ for shot 12218 .

Fig. 2.15 (a) The axial magnetic field at the outer electrode vs time is shown at various axial locations for shot 5877 . Each trace is offset vertically by an distance proportional to the axial location. (b) Same for shot 5333.

Fig. 2.16 Schematic of RACE bakeable electrodes.

Fig. 2.17 Velocity of CT's in RACE as calculated from the transit time between chordal-view interferometers at $\mathrm{z}=50 \mathrm{~cm}$ and $\mathrm{z}=124 \mathrm{~cm}$ in the bakeable electrode section. The velocity is plotted vs. shot number with different markers denoting gun/accelerator bank voltage and electrode condition.

Fig. 2.18 Trajectory (axial distance vs. time) of the CT for RACE shot 15020. Each marker shows the time of peak signal at different diagnostics located along the accelerator. The maximum velocity apparently exceeds $50 \mathrm{~cm} / \mu \mathrm{s}$.

Fig. 2.19 Interelectrode region divided into low density channel( region 2) and dense wall plasma (region 1 ).

Fig. 3.1 Schematic MARAUDER configuration.

Fig. 3.2 Geometry of 16 channel x-ray photodiode detector array.

Fig. 3.3 Power density of the emitted VUV radiation in kilowatts $/ \mathrm{cm}^{-3}$ as a function of radius and time for a MARAUDER shot. 
Fig. 3.4 CT and electrode geometry for TRAC2 simulations of a "gentle comer" vs "sharp comer" transition in the MARAUDER accelerator.

Fig. 3.5 Lasnex predictions for $\mathrm{x}$-ray output in different spectral bins on MARAUDER and a $50 \mathrm{MJ}$ accelerator. Comp. is the compression ratio.

Fig. 3.6 Lasnex output spectra for different CT parameters

Fig. 4.1 (a) Circuit and (b) geometry for MARAUDER-driven radial CT acclelerator.

Fig. 4.2 Long-pulse, sustained CT acclerator

Fig. 4.3 Accelerator current and CT magnetic flux waveforms for long-pulse, sustained CT accelerator. 


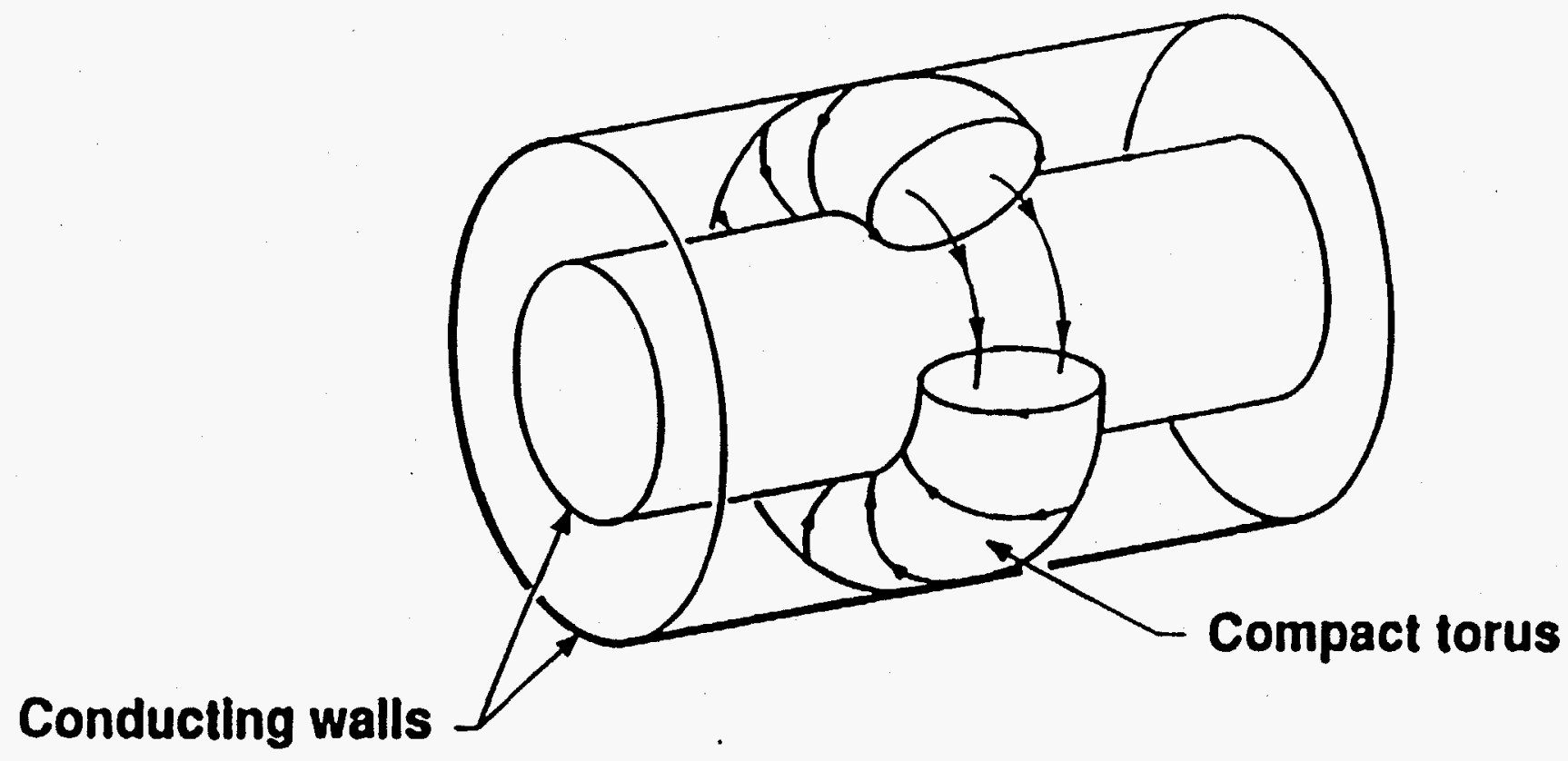

- it is maintained in radial equilibrium by an external field or by induced currents in conducting walls

- the compact torus is a state of low free energy with observed rugged stability and long lifetime

Fig. 1.1 


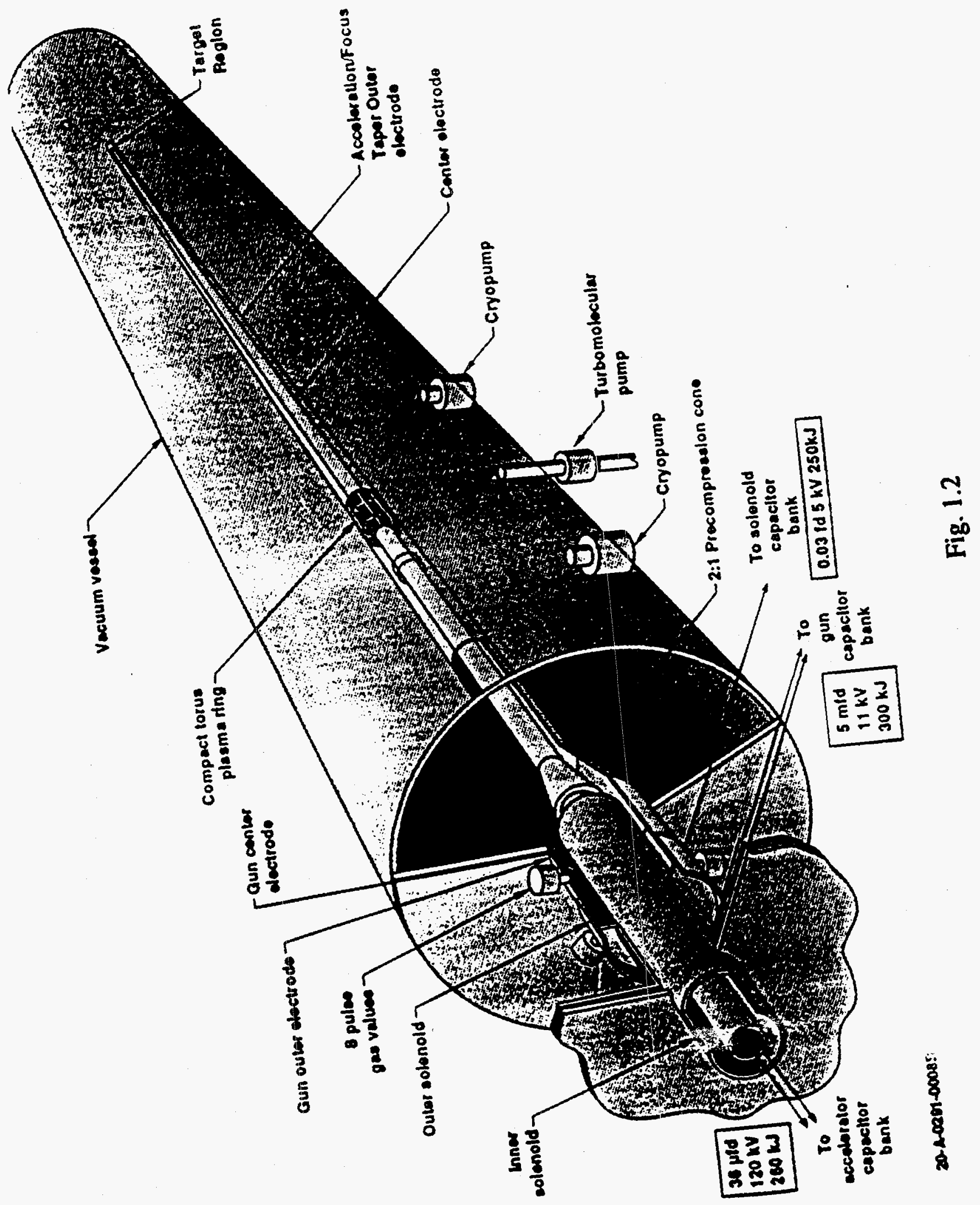




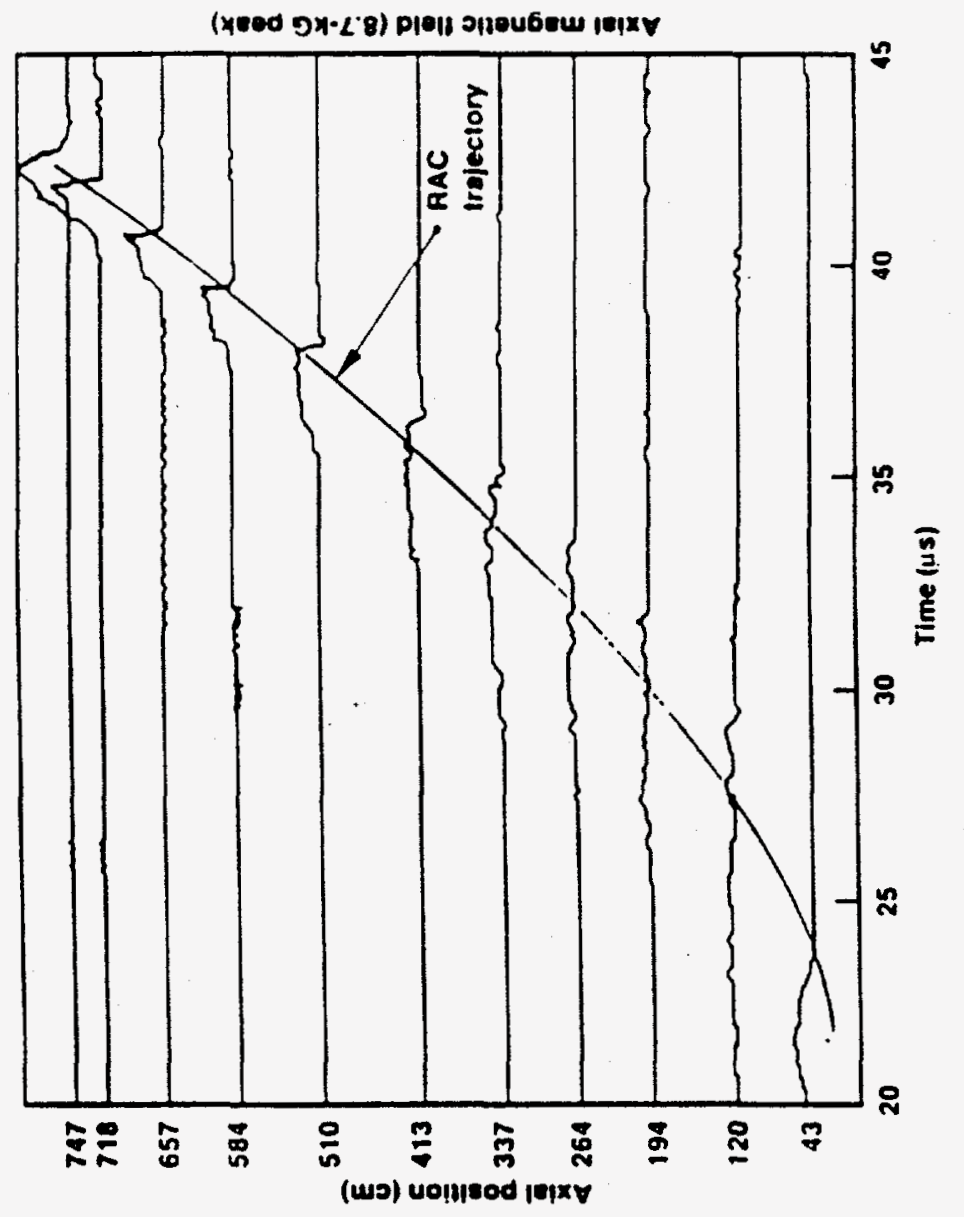

m 


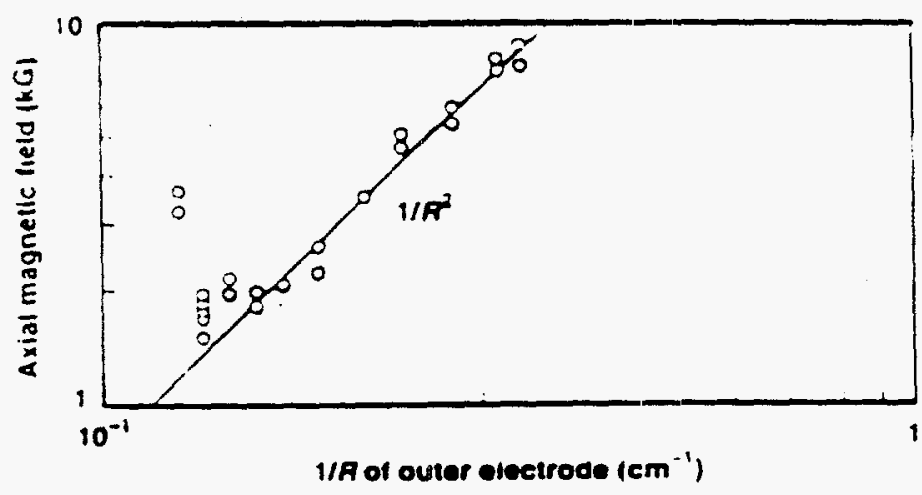

.1 .4

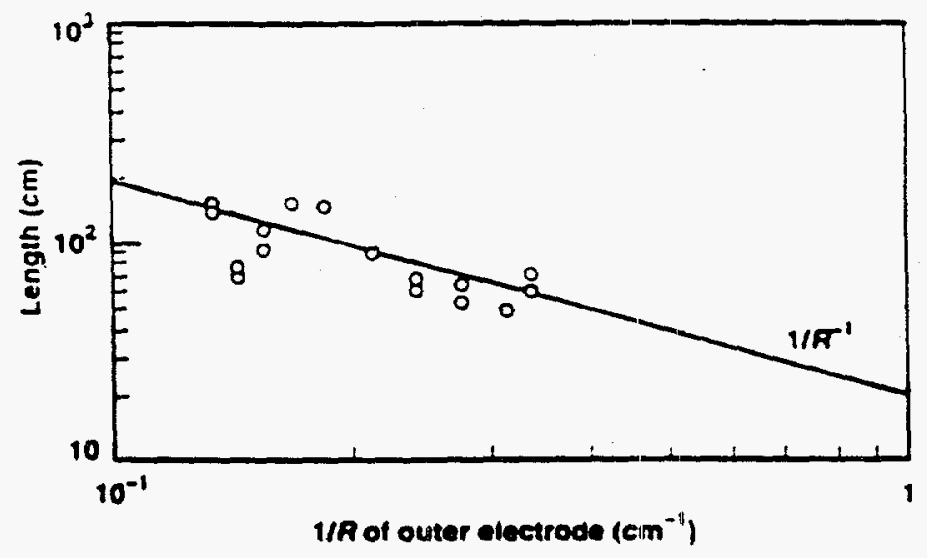

Fig. 1.5a

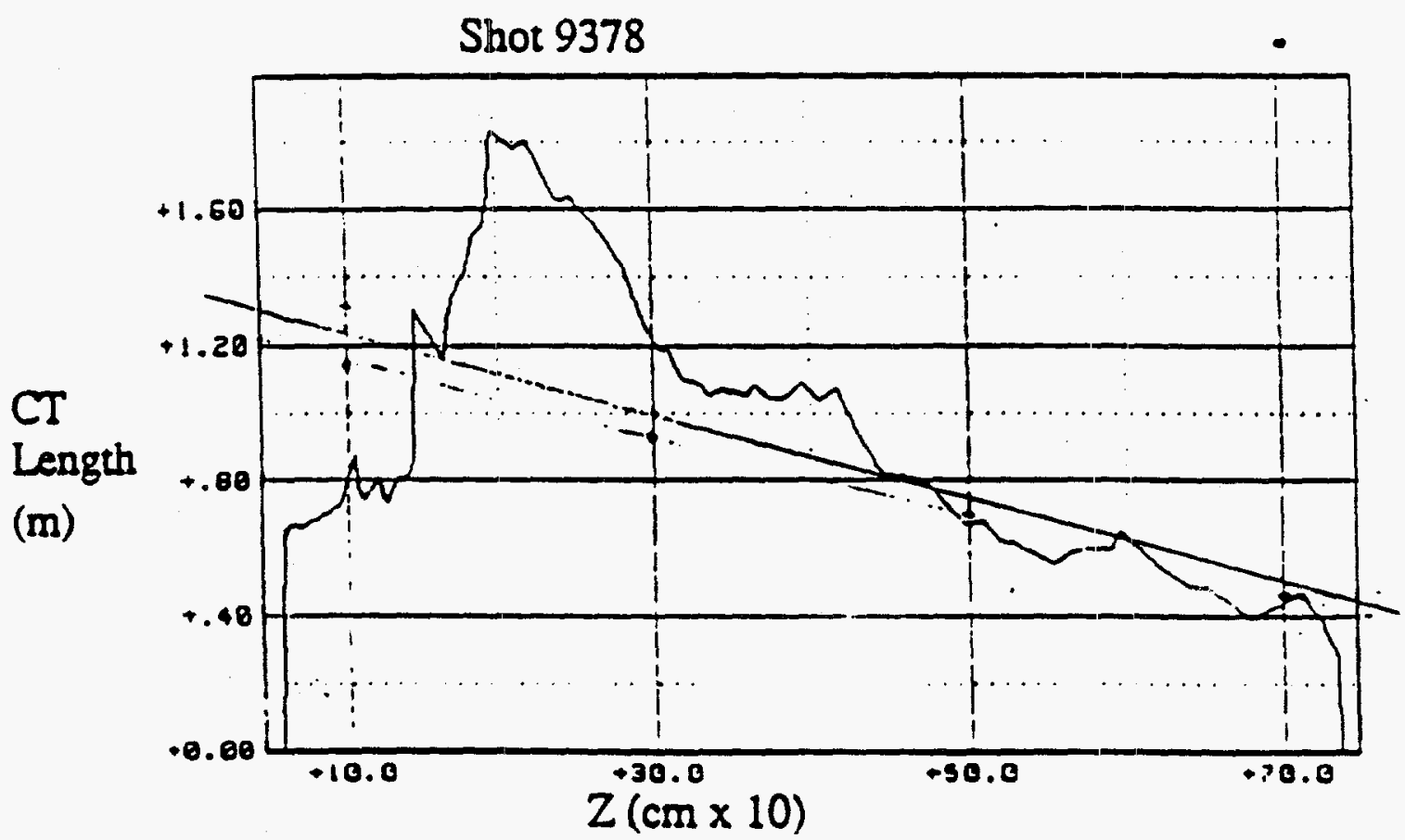

g. $1.5 b$ 


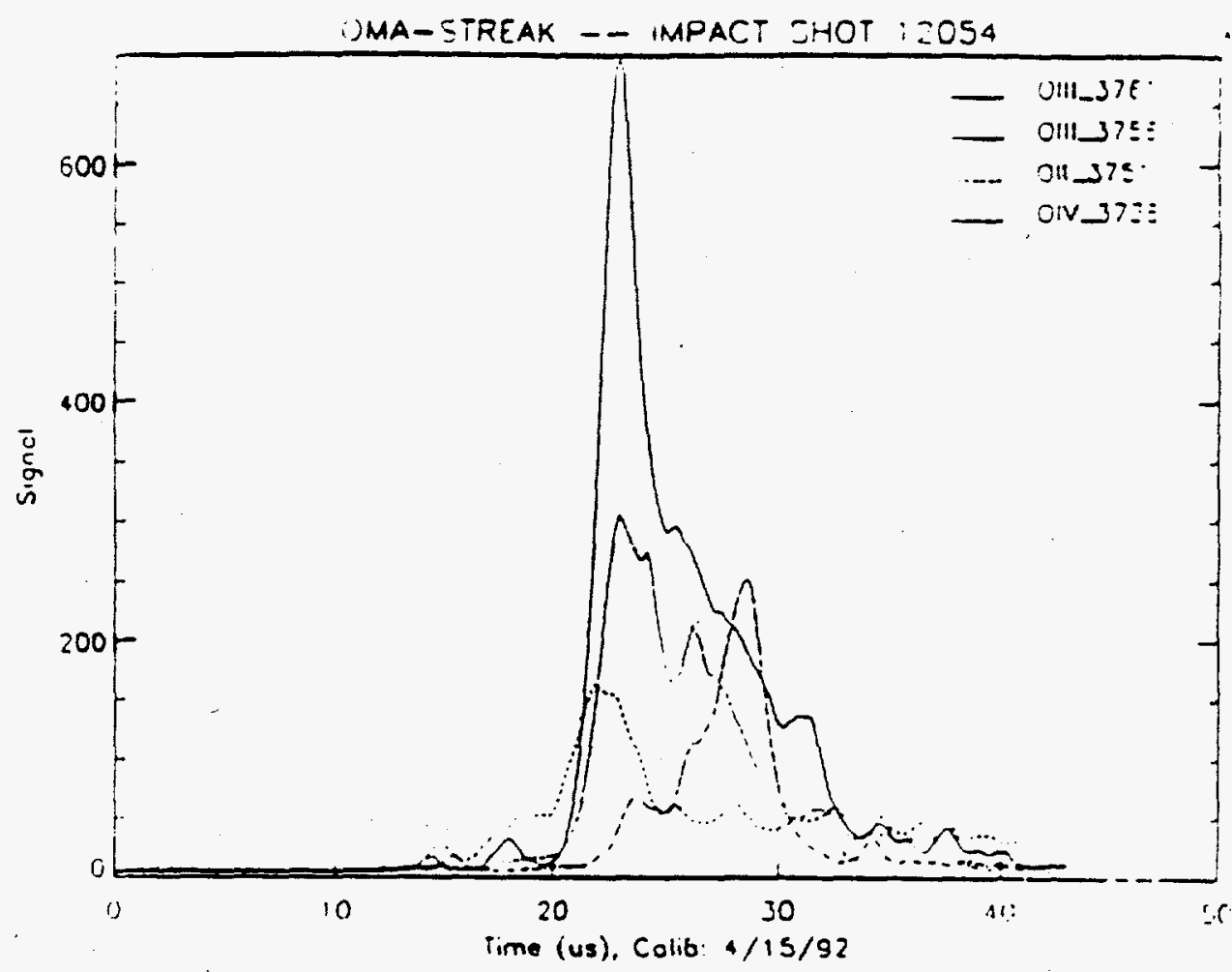

Fig. 2.1a

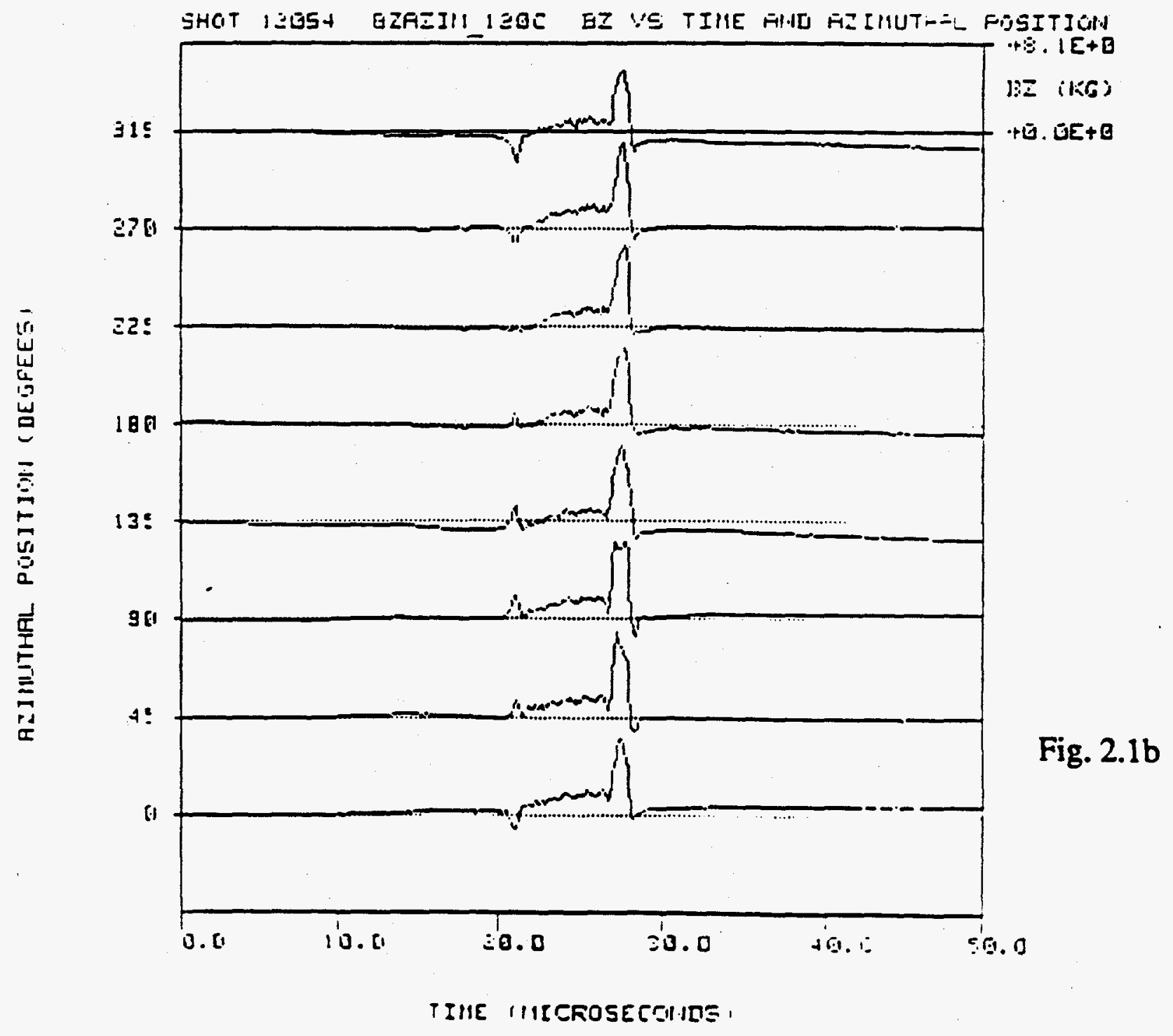


NE_Z_E393.1 CH-RVG WE RT $194 \mathrm{~cm}$ FIND EZ FT $194 \mathrm{~cm}$ VS TIME 5HOT ZOS4

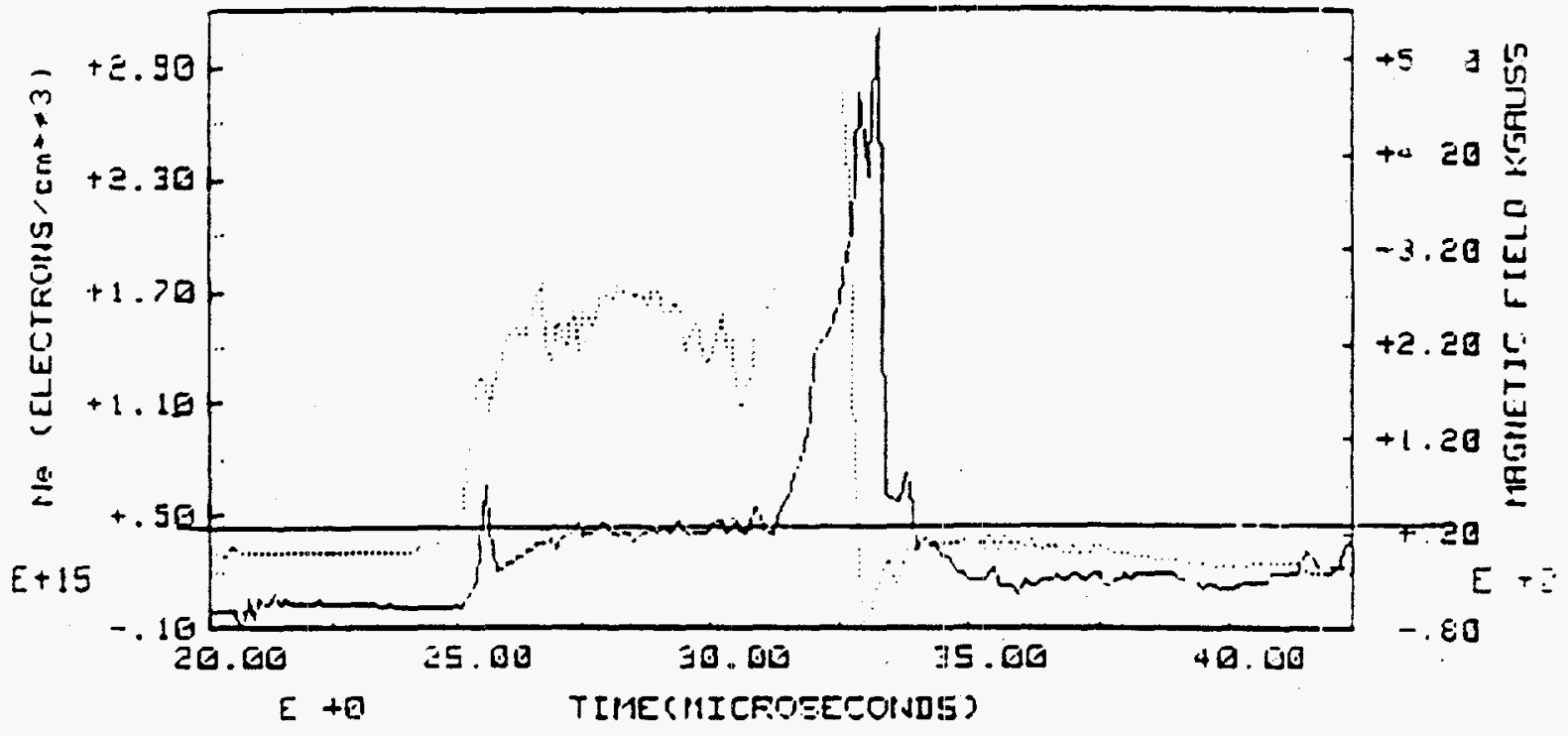

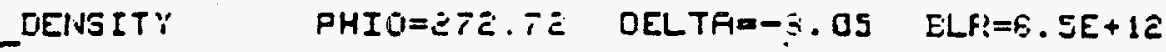

......MAG FIELD FWM/2 TWAZ=33.11 FWM/2 TBAS.=31.32

TIME SMOOTHIHG FACTORS: DENSITY-G E FIELU-G

Fig. 2.2a

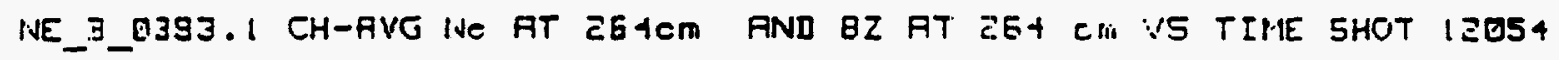

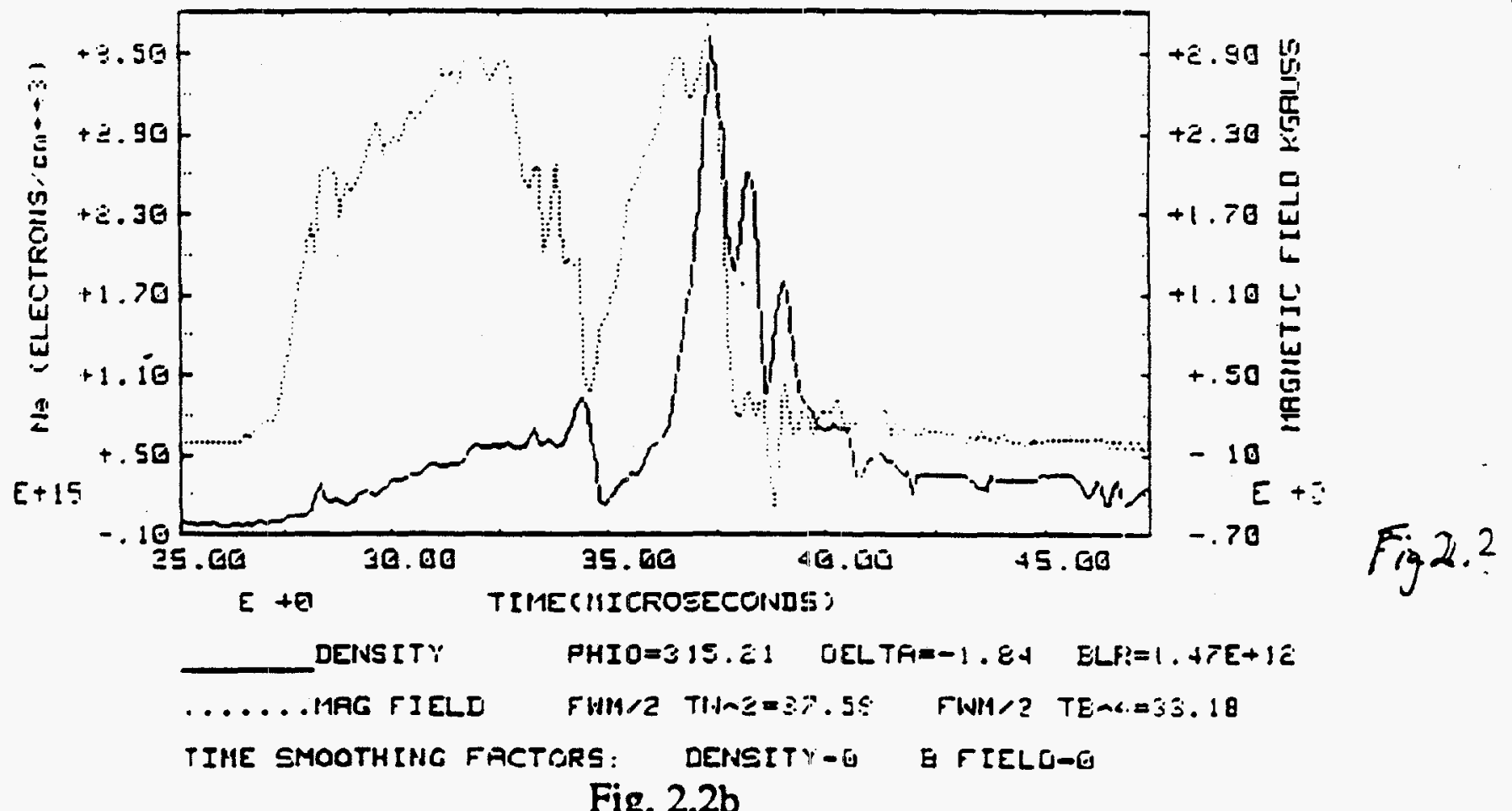

Fig. 2.2b 


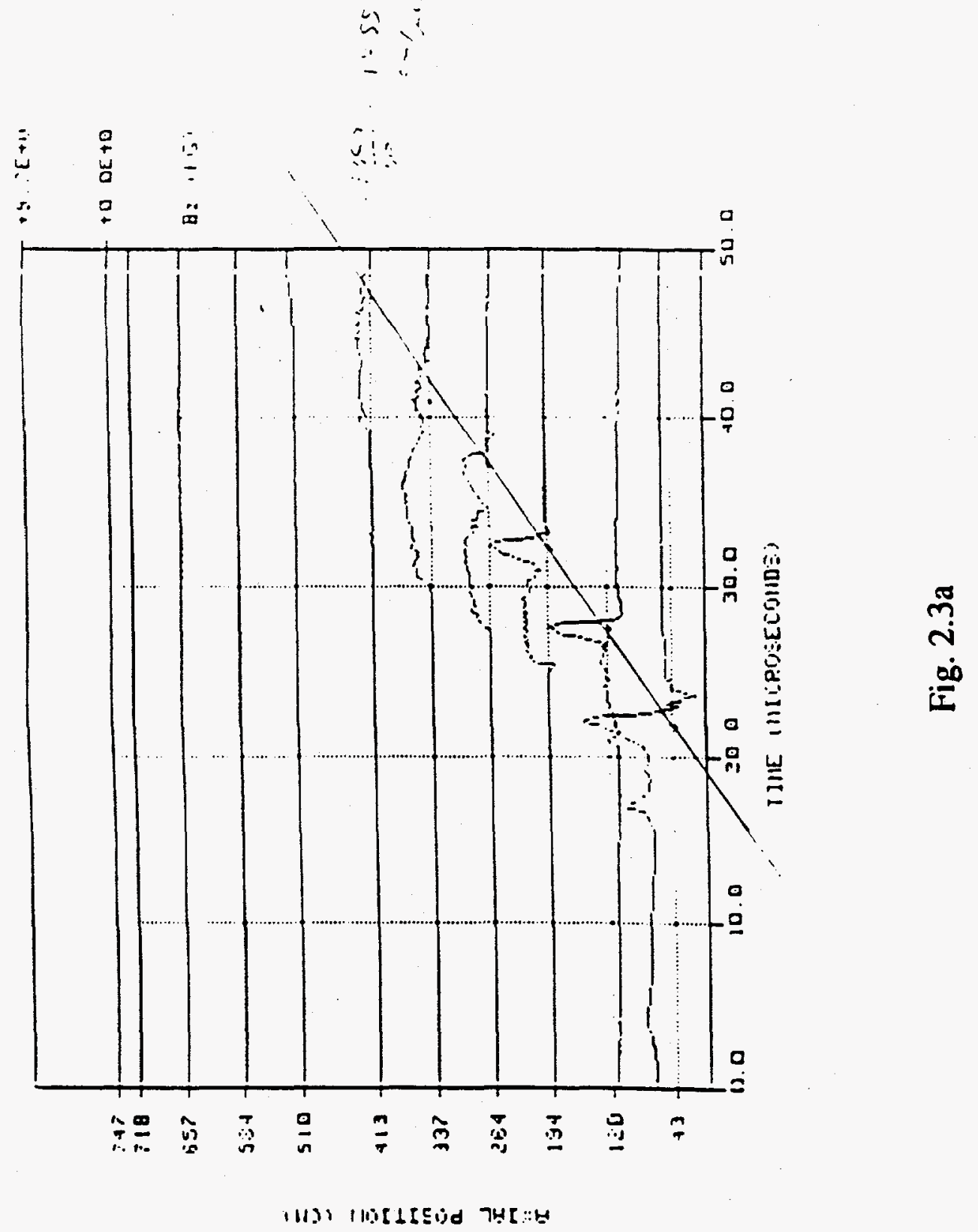




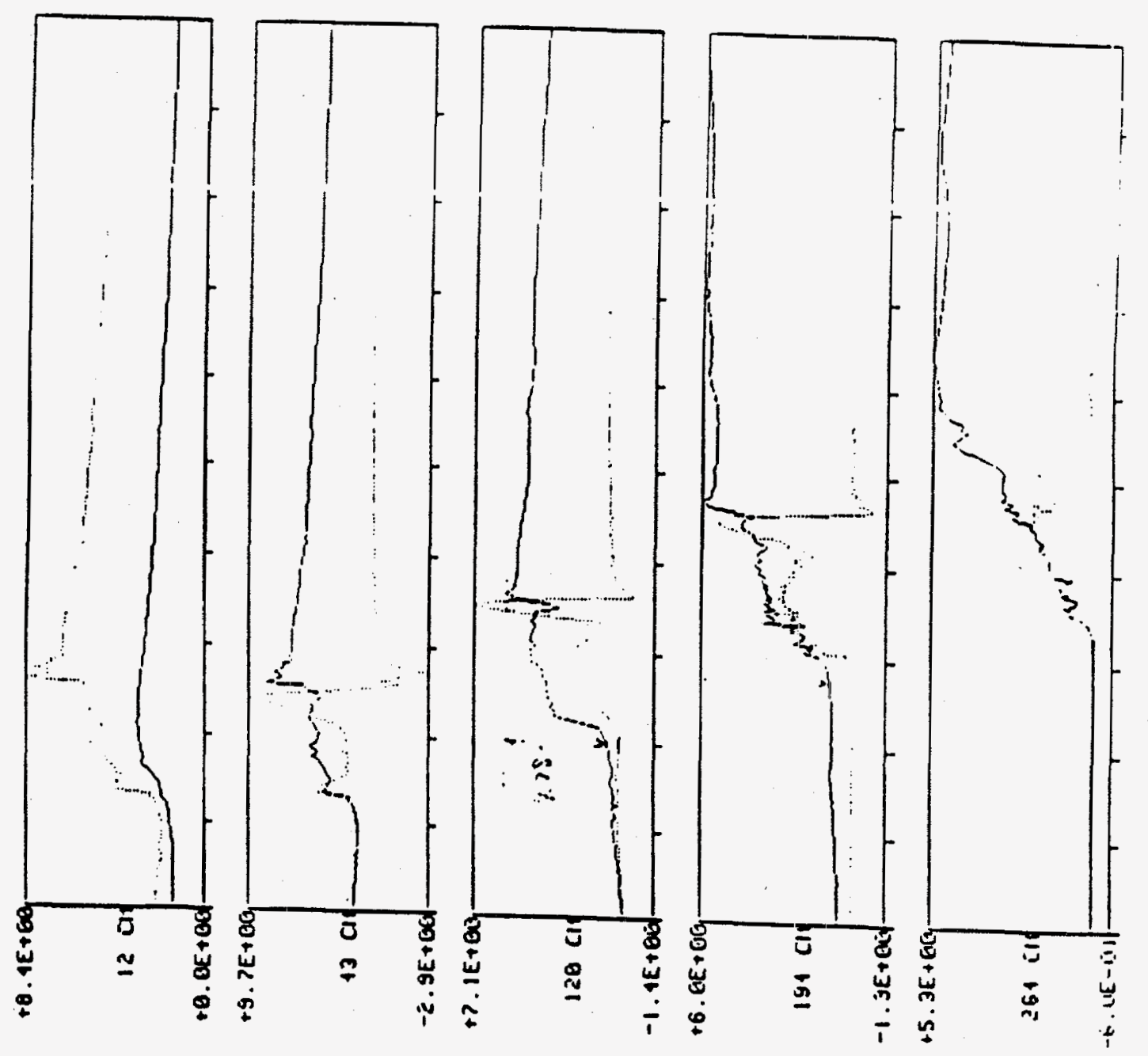

ติ 
Density of front of CT similar 10 neutral density required to provide constant velocity by momentum input in RACE (Long cone)

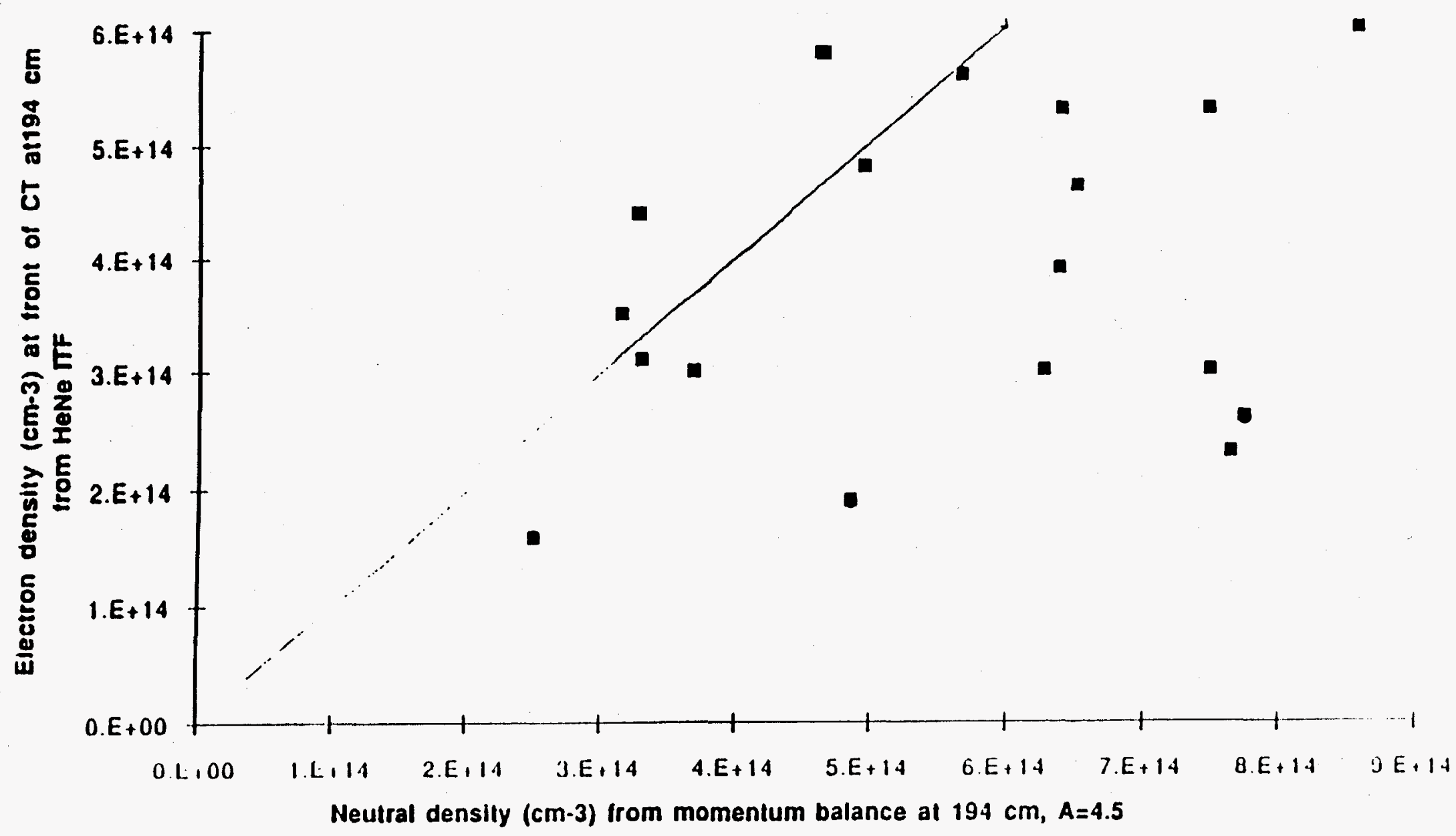

Fig. 2.4 


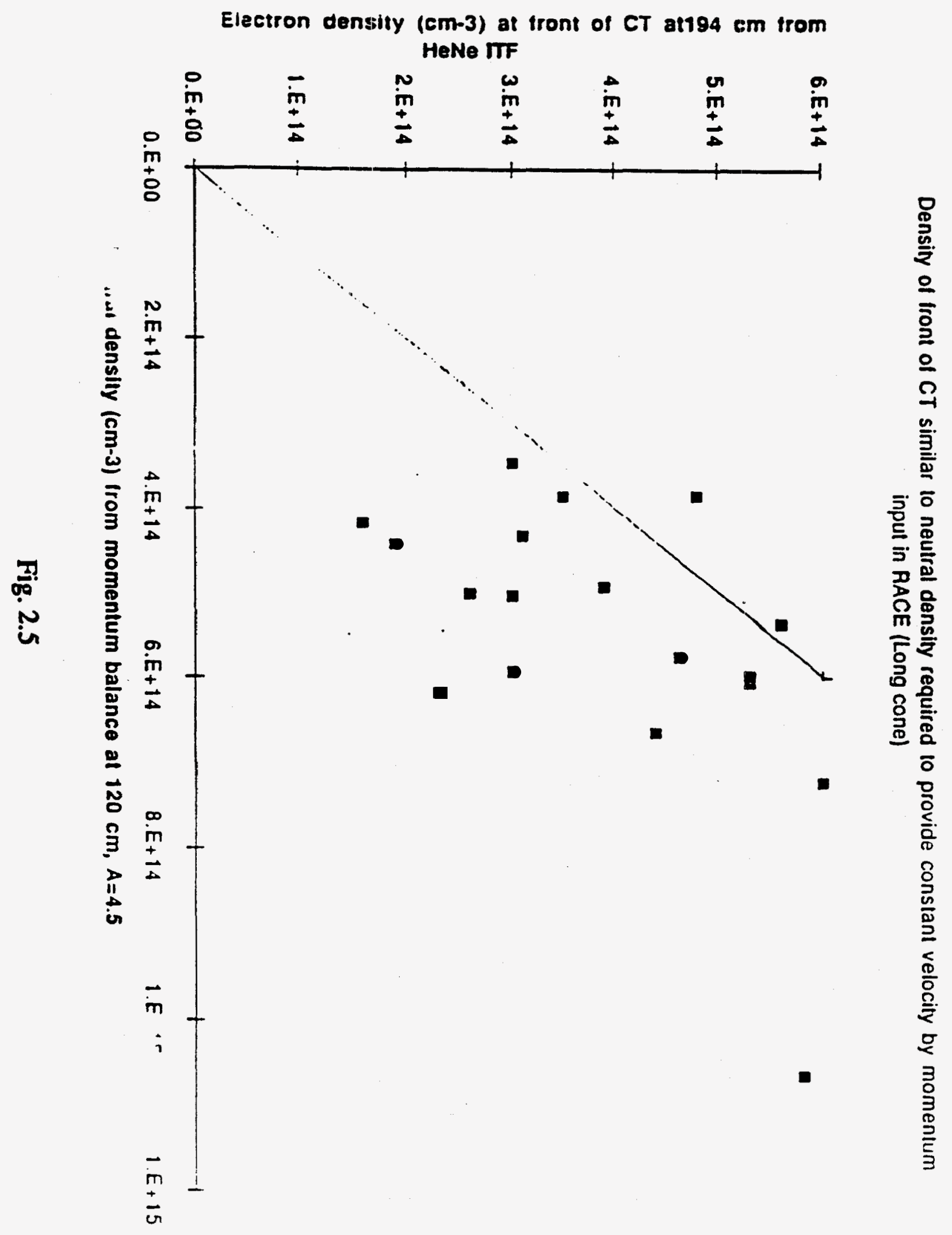




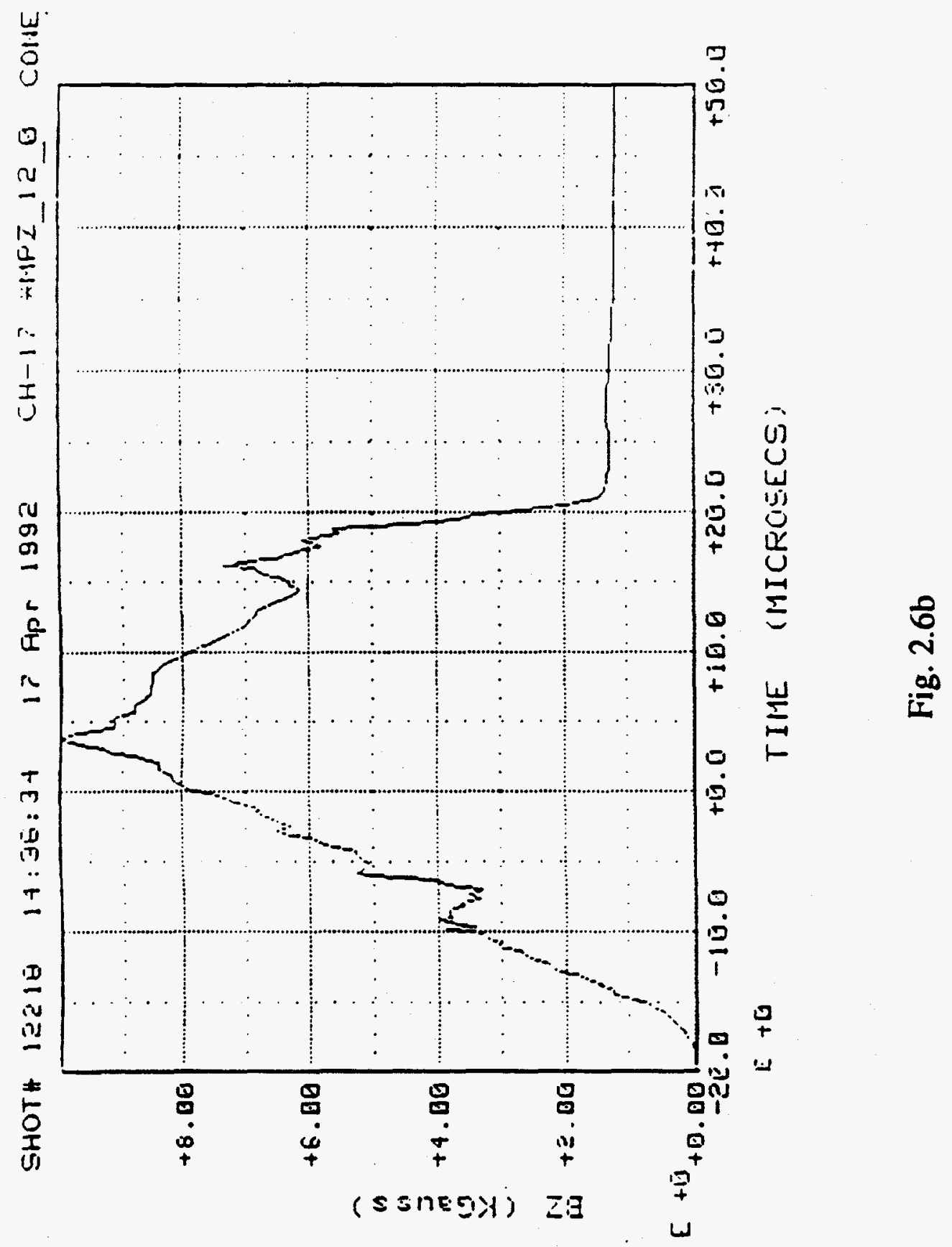




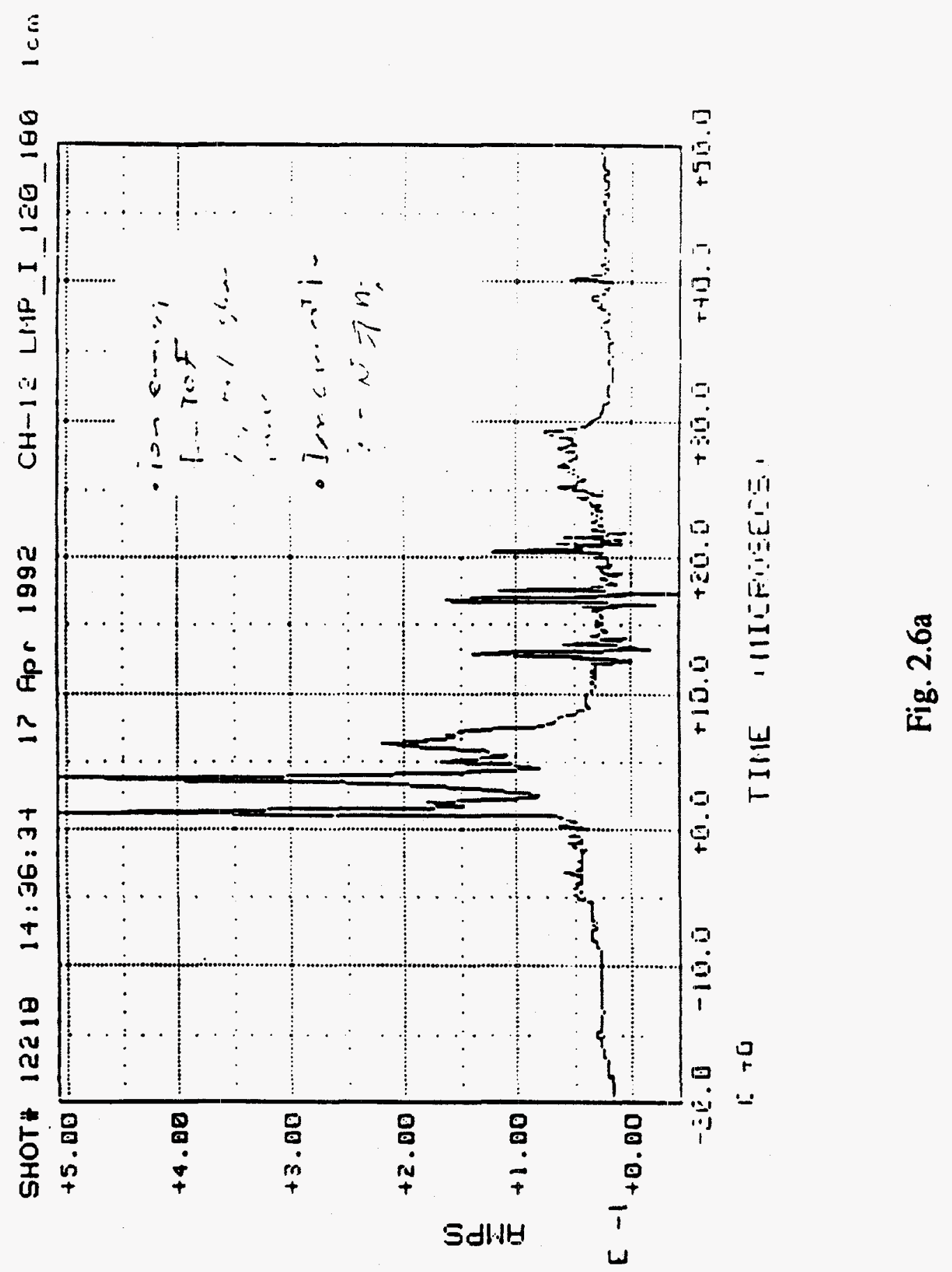




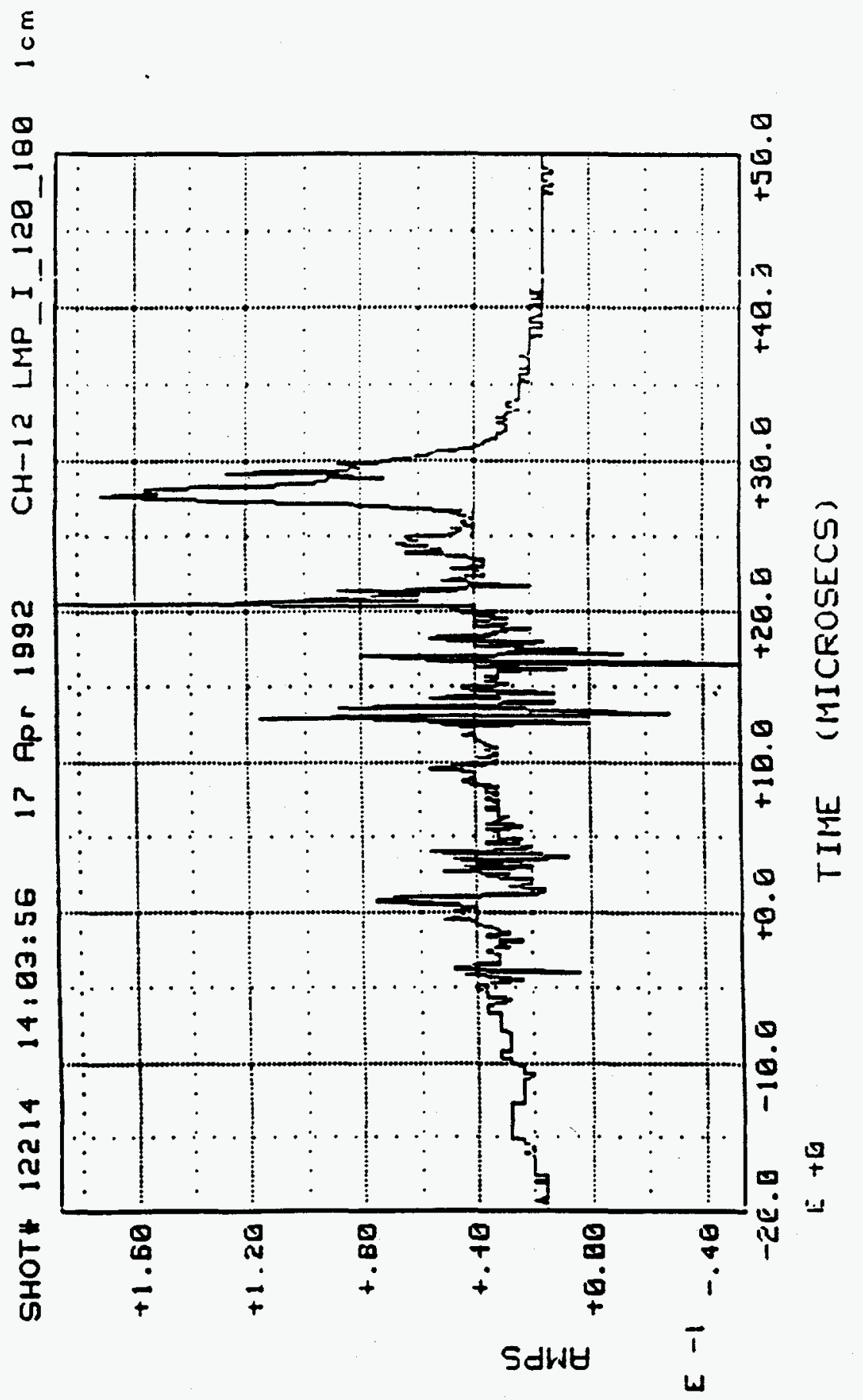

iv 


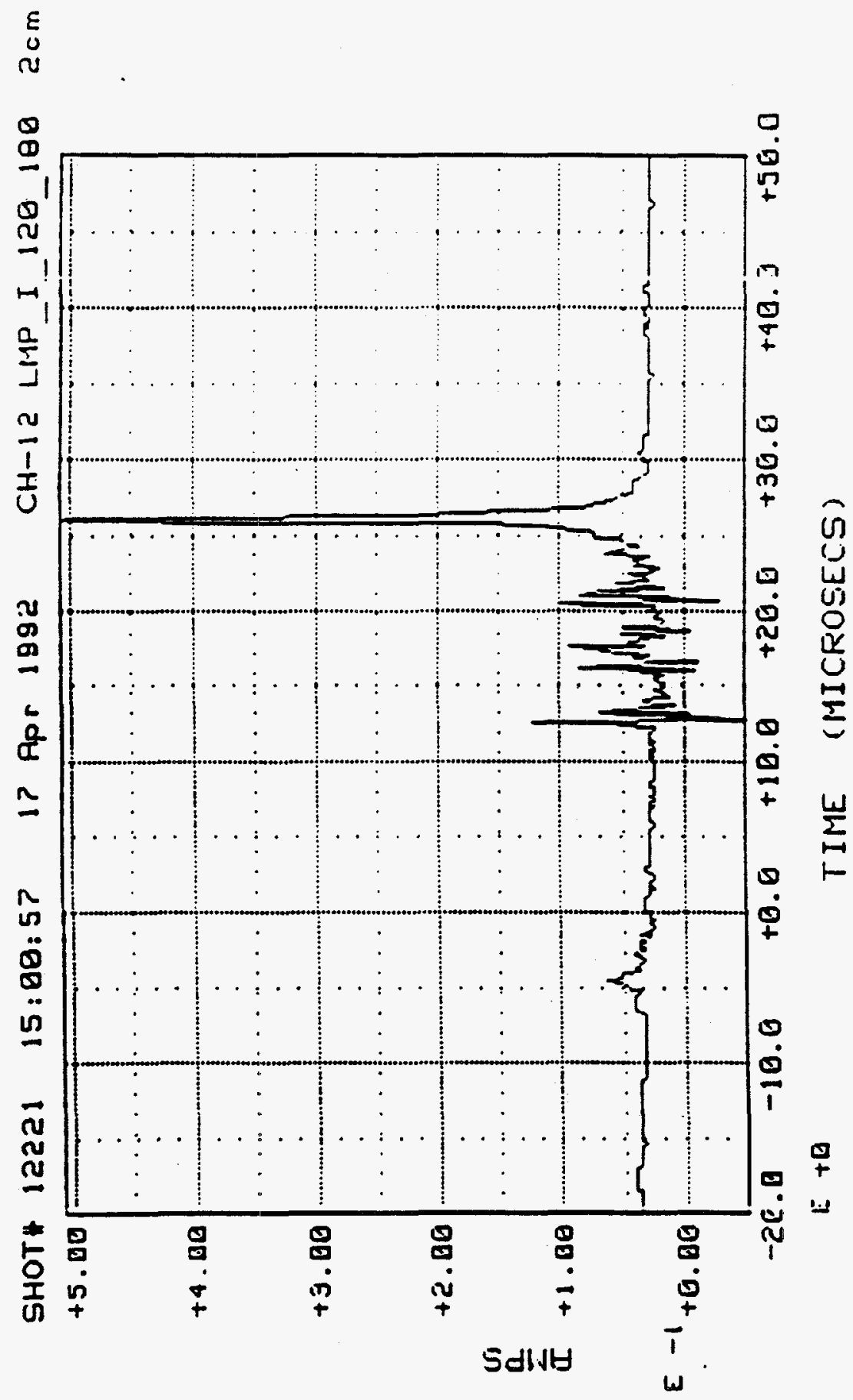

$\underset{i}{\infty}$ 
$\underset{\substack{n \\ 0}}{0}$

$\stackrel{\infty}{\sim}$

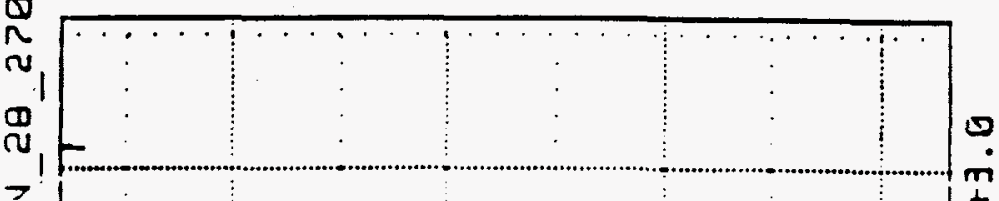

길

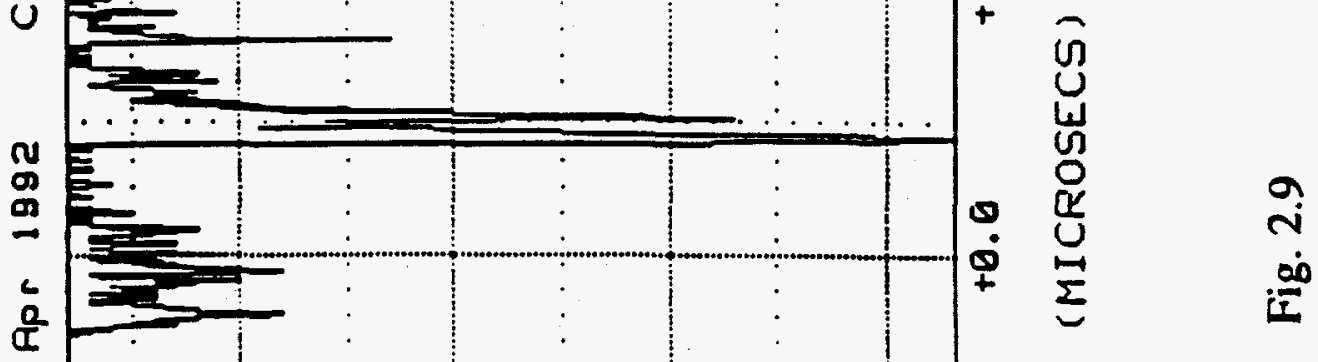

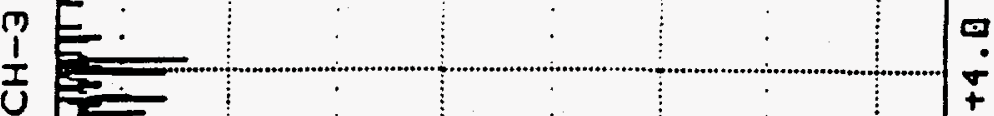
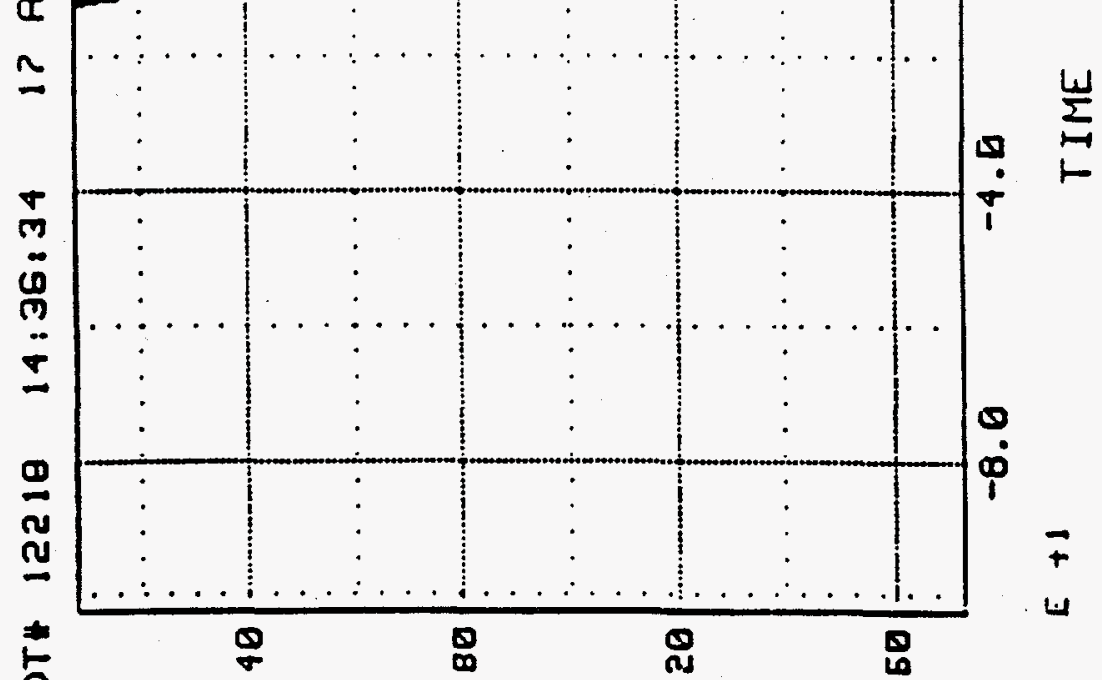

点 $\quad \frac{0}{5} \quad$ i

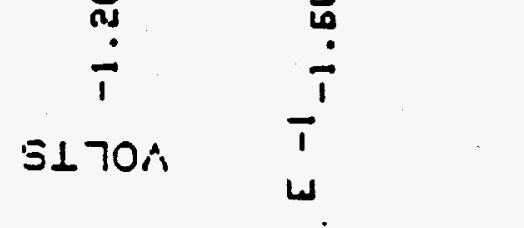




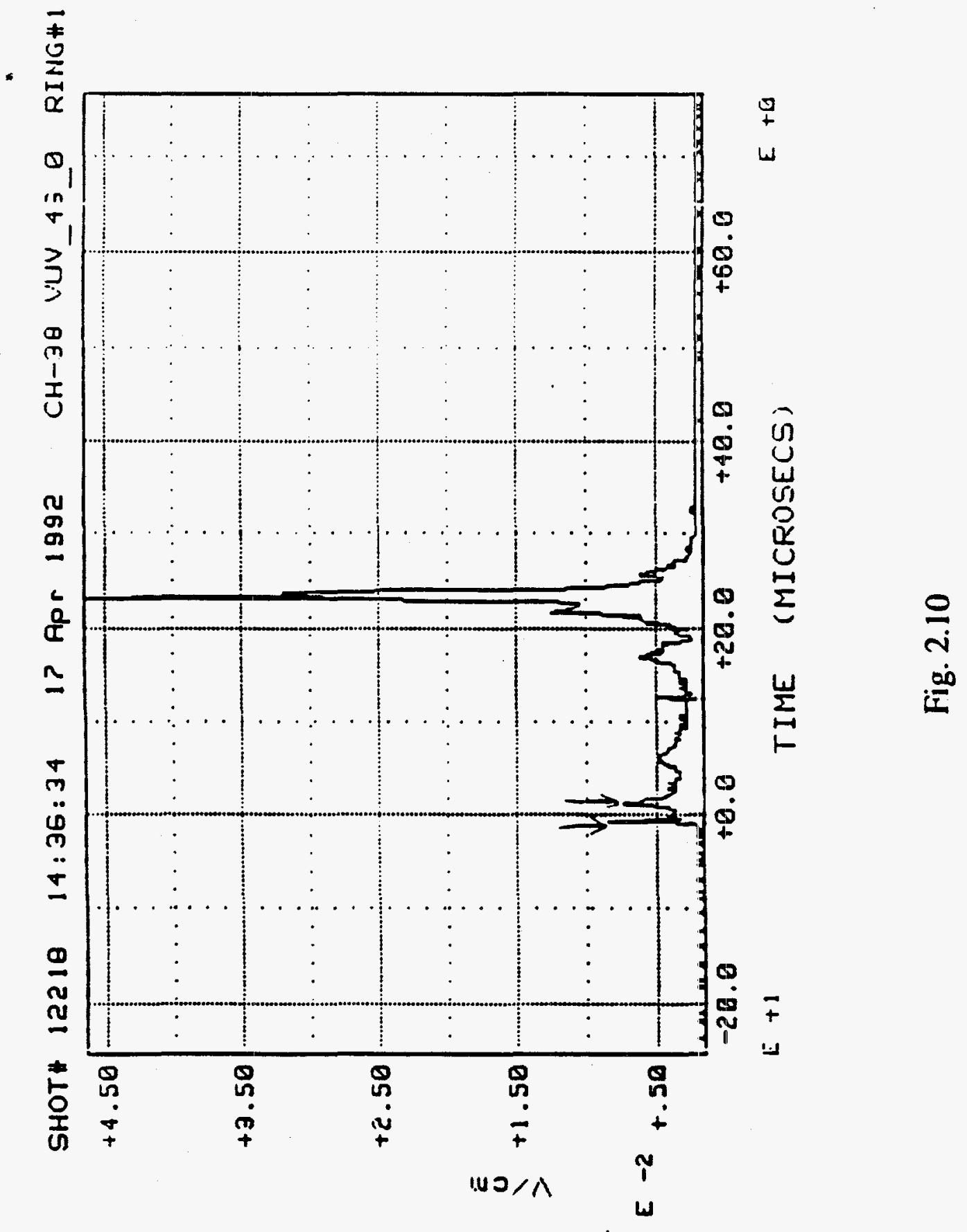




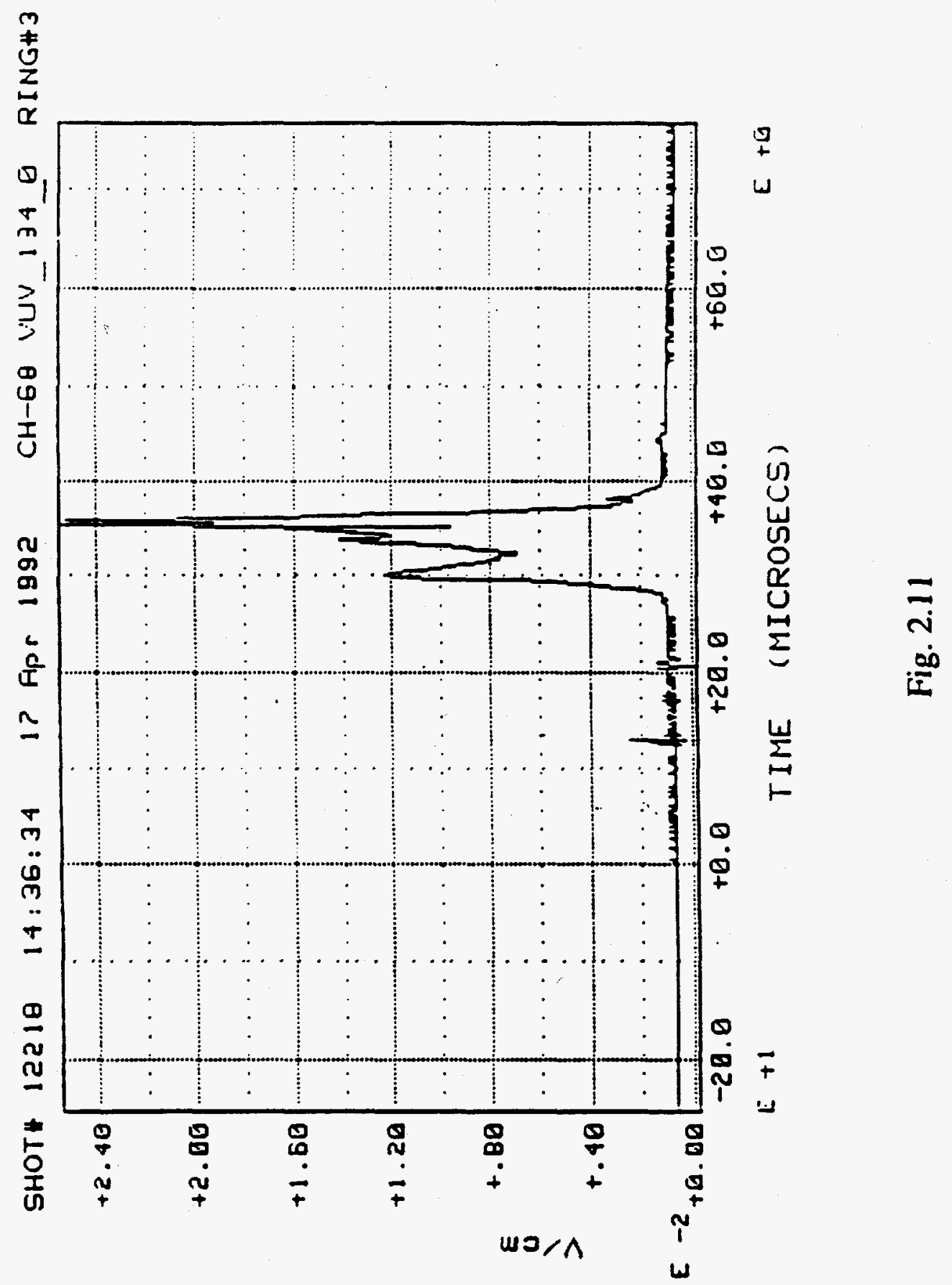




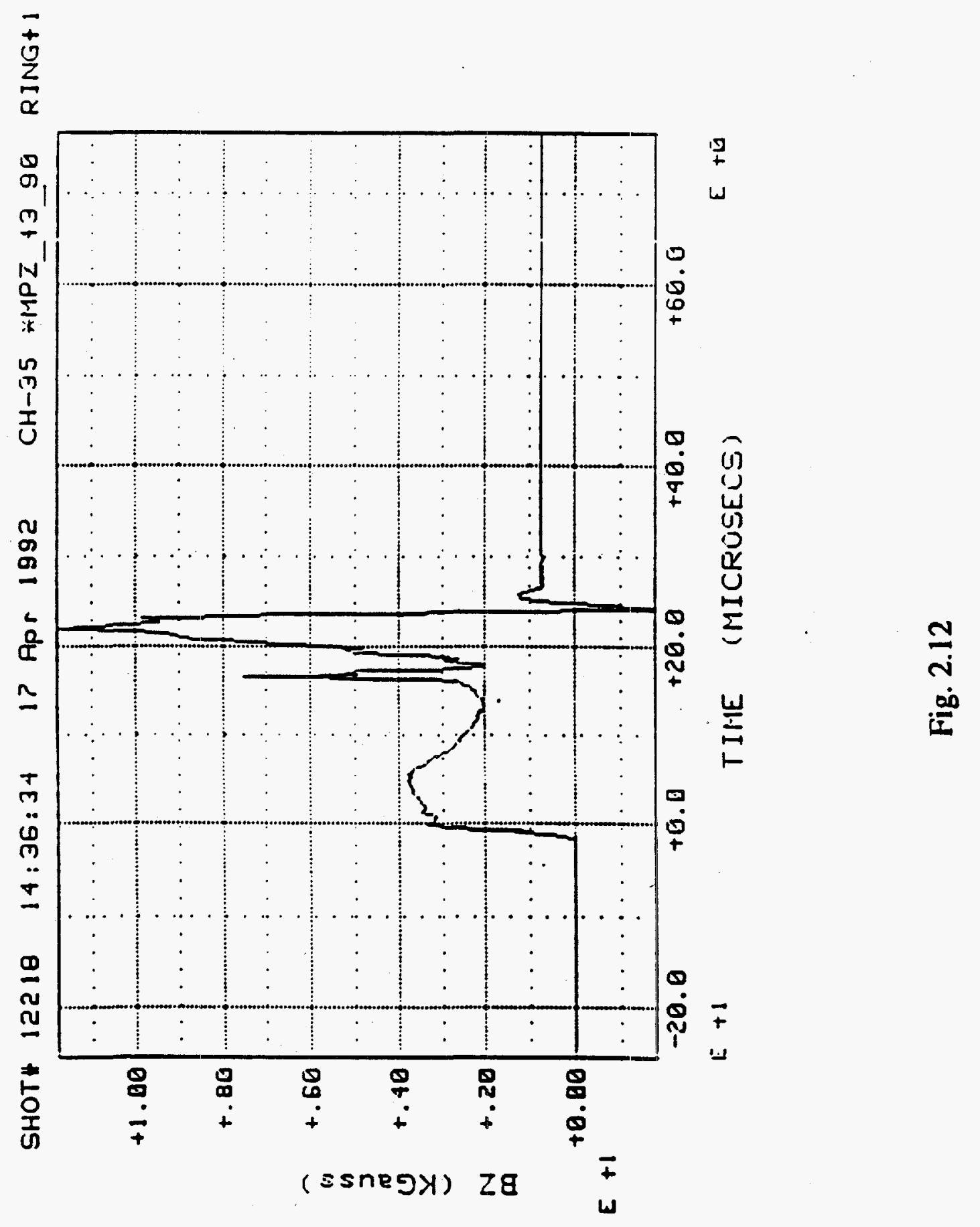


$\underset{\sim}{*}$

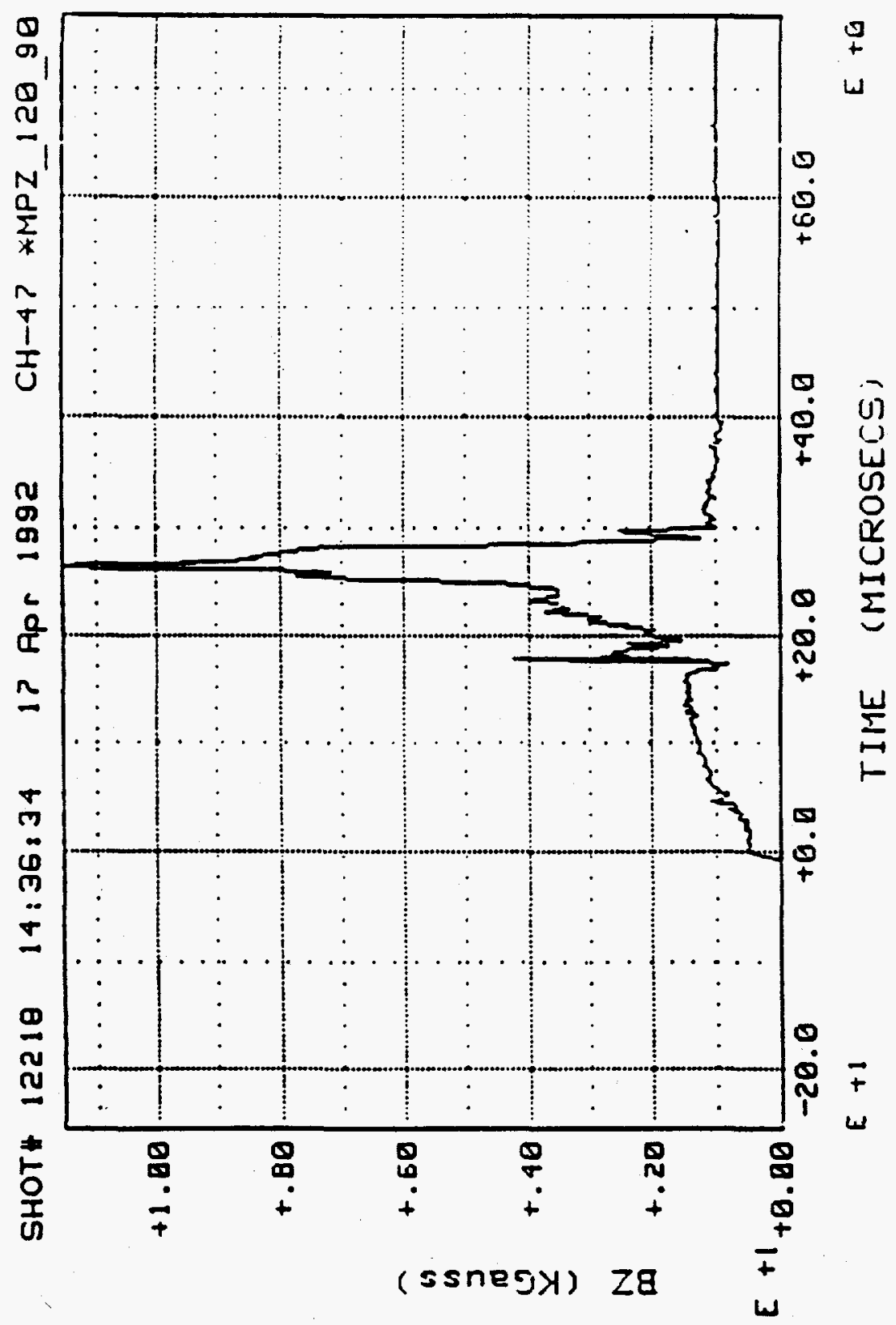

$\frac{m}{n}$ 


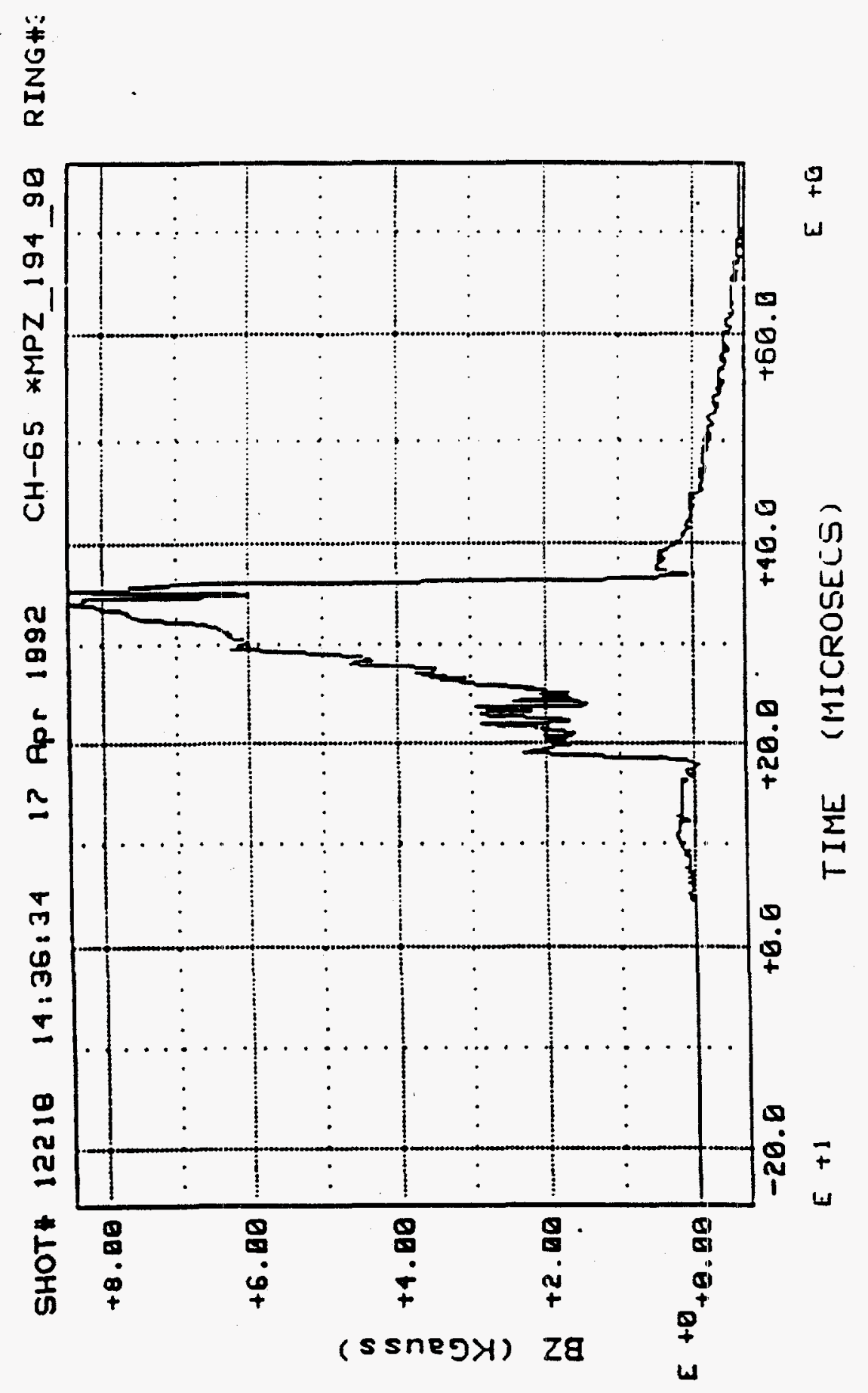

$\frac{a}{i}$ 


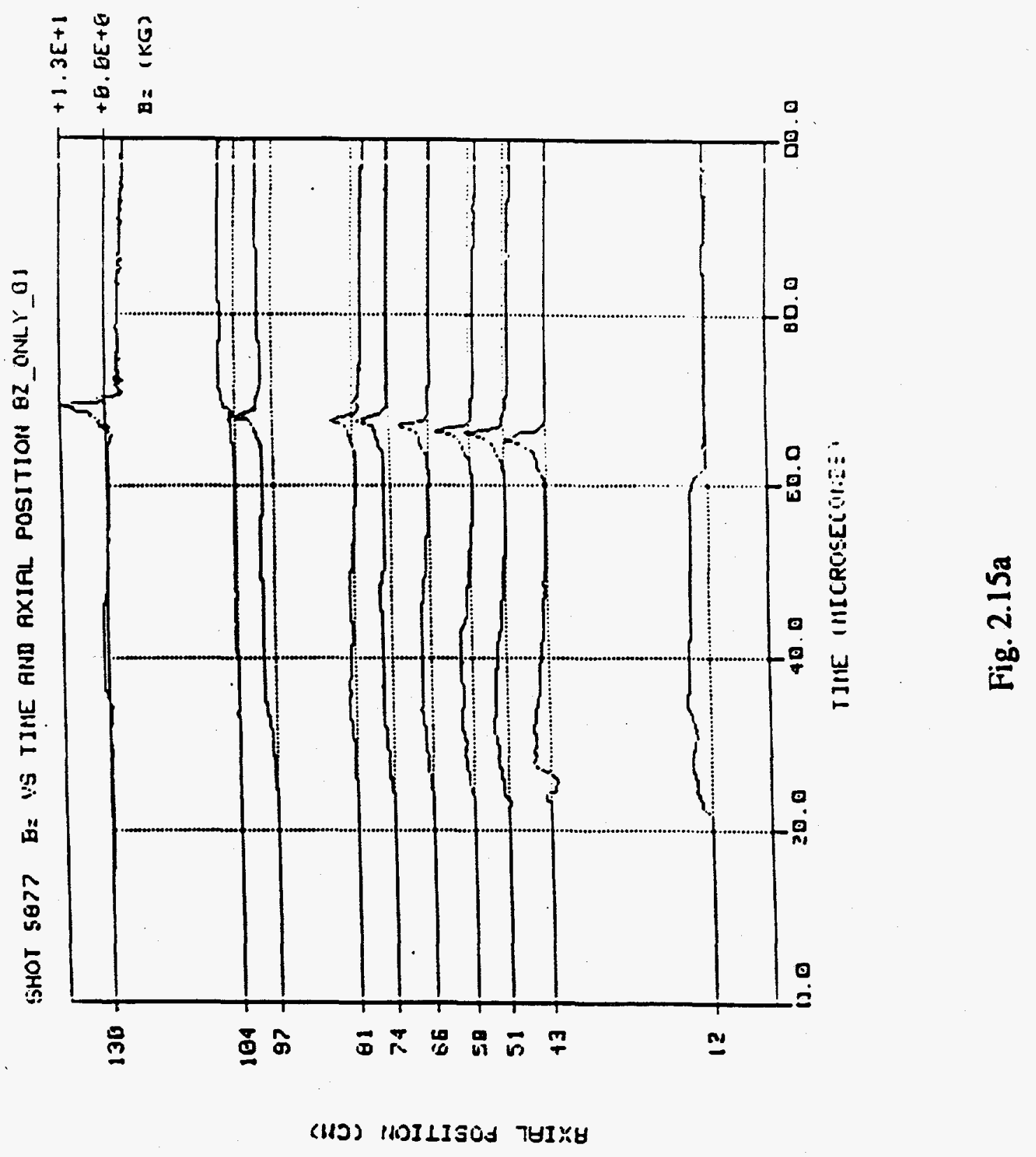




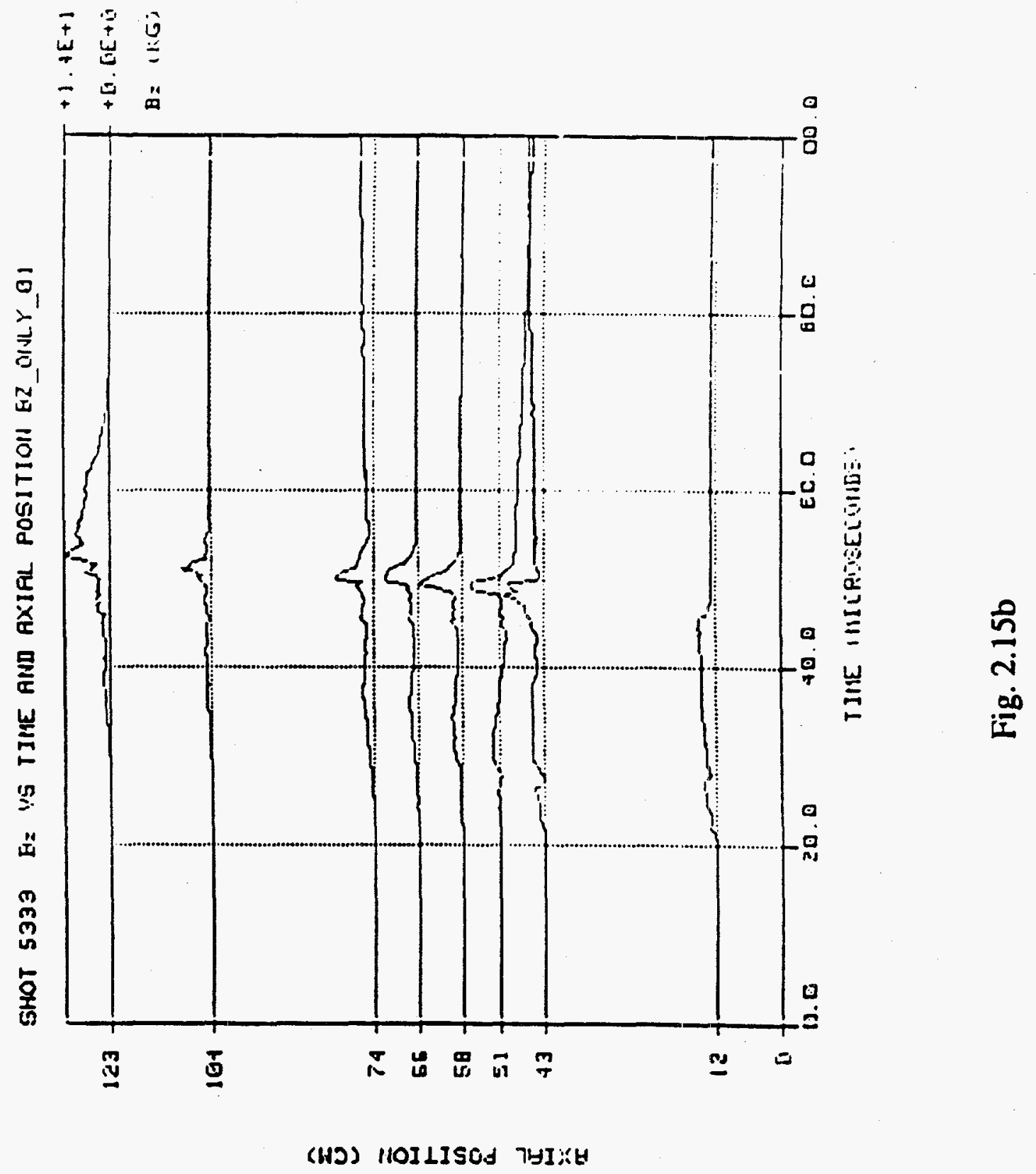



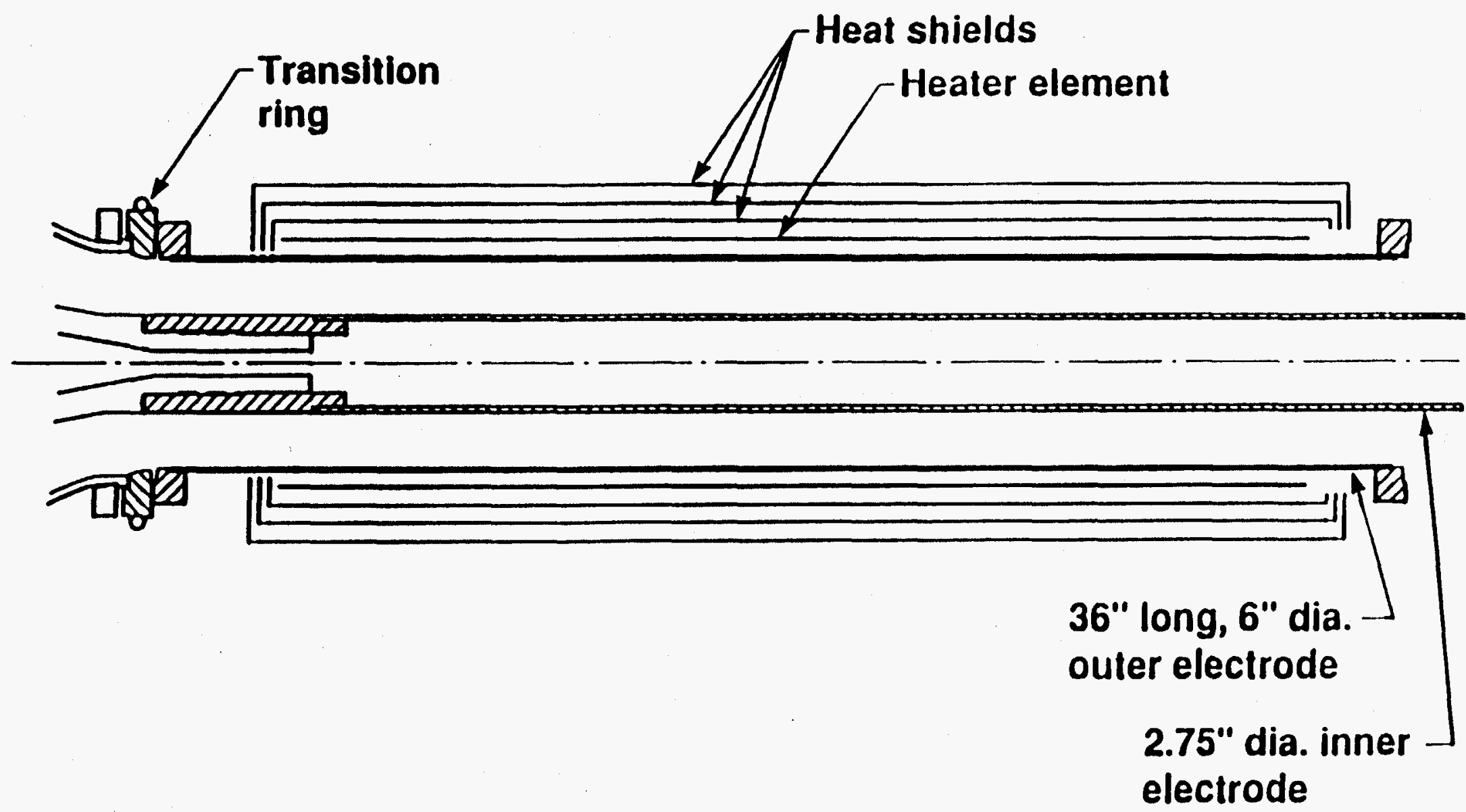

Fig. 2.16 


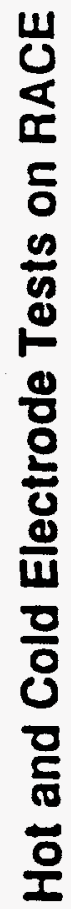

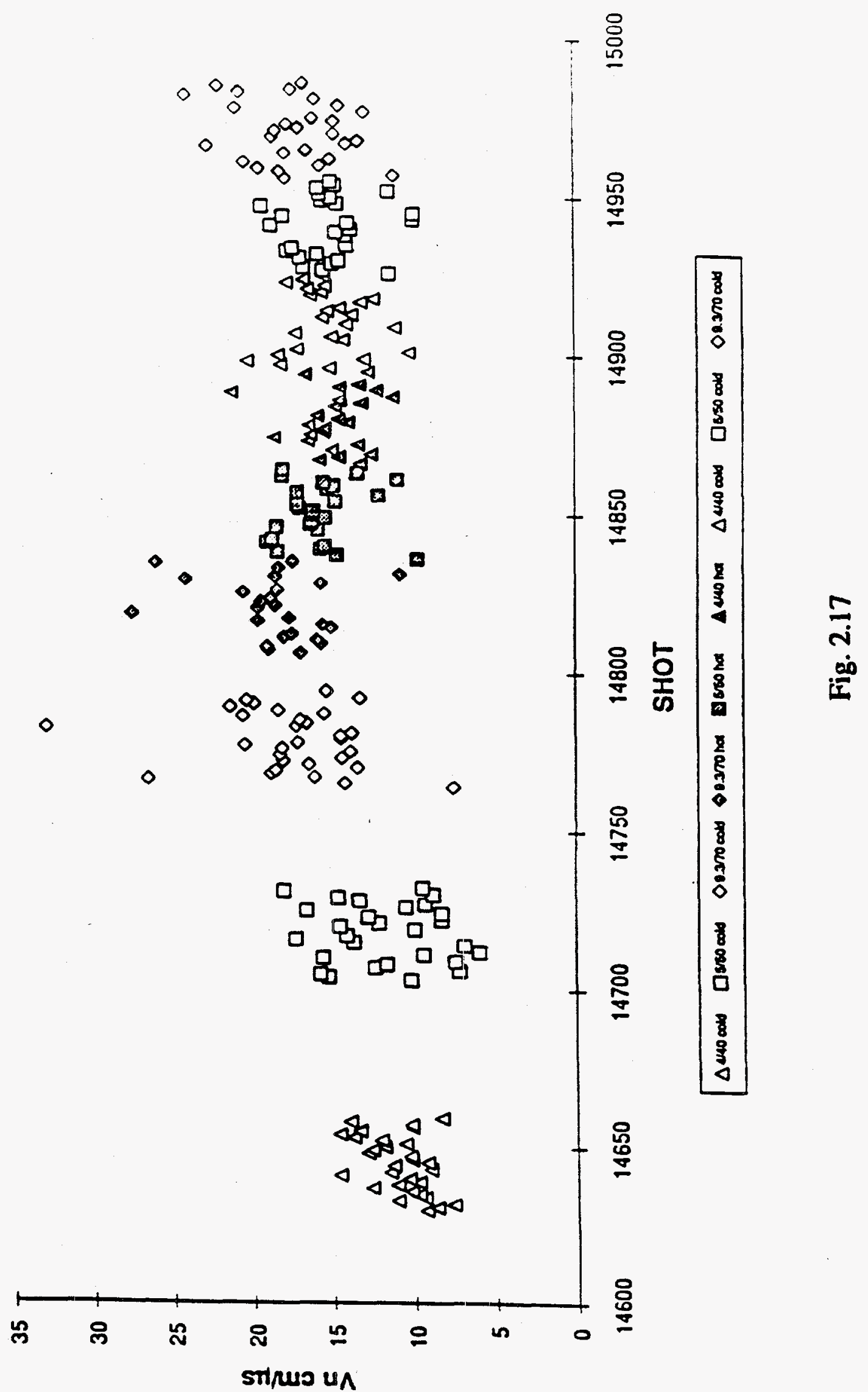


Shot 15020

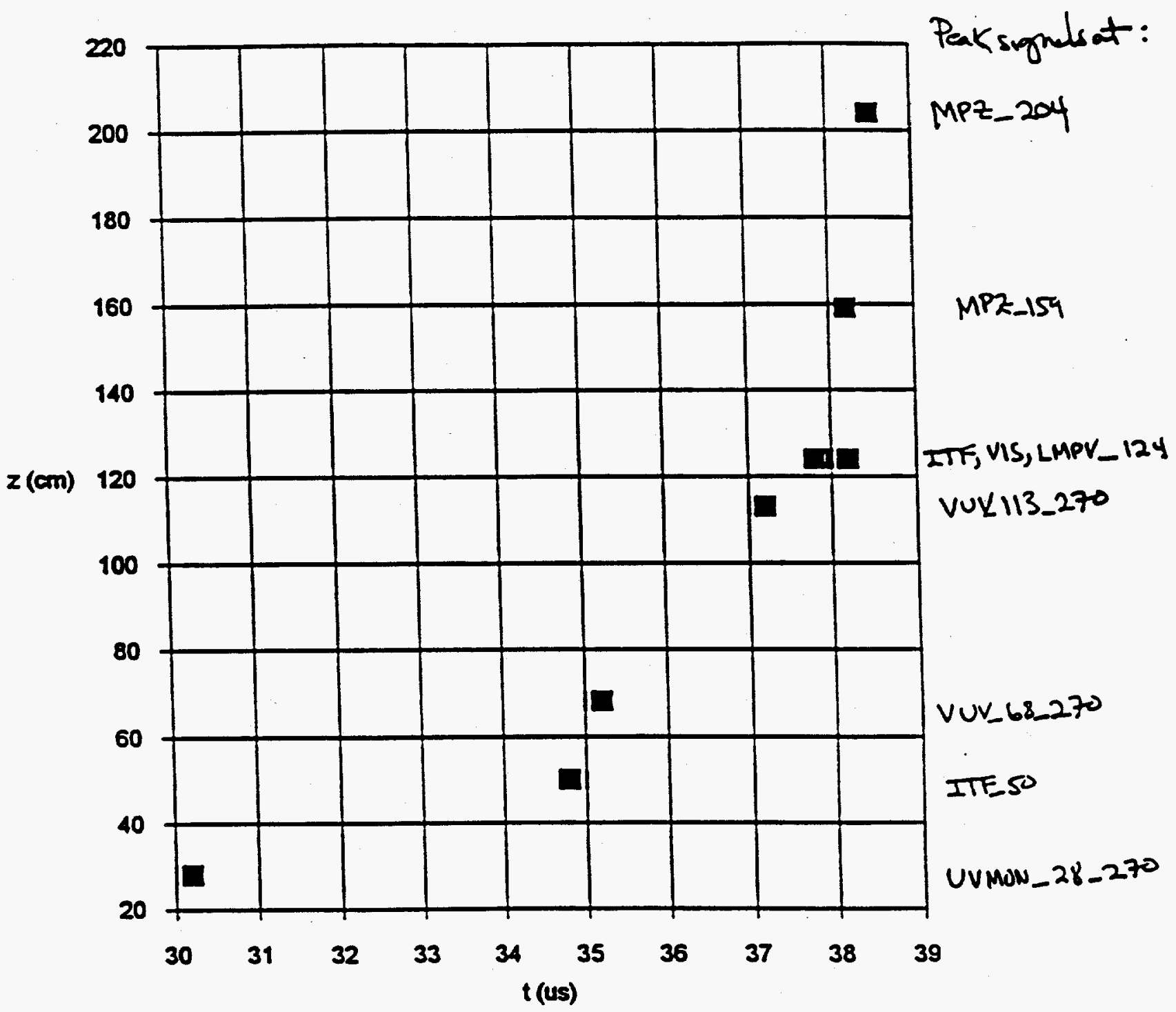

Fig. 2.18 


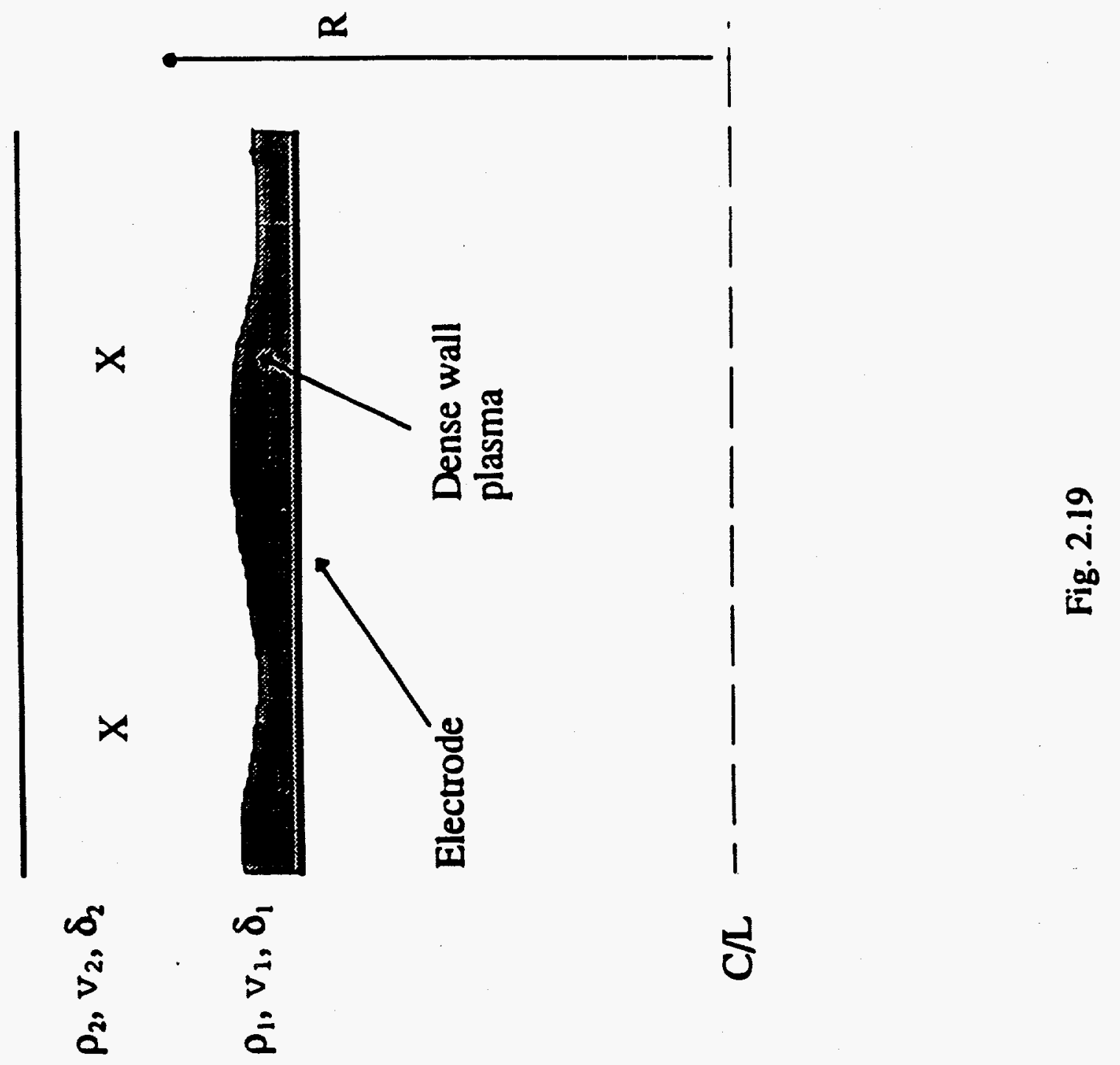




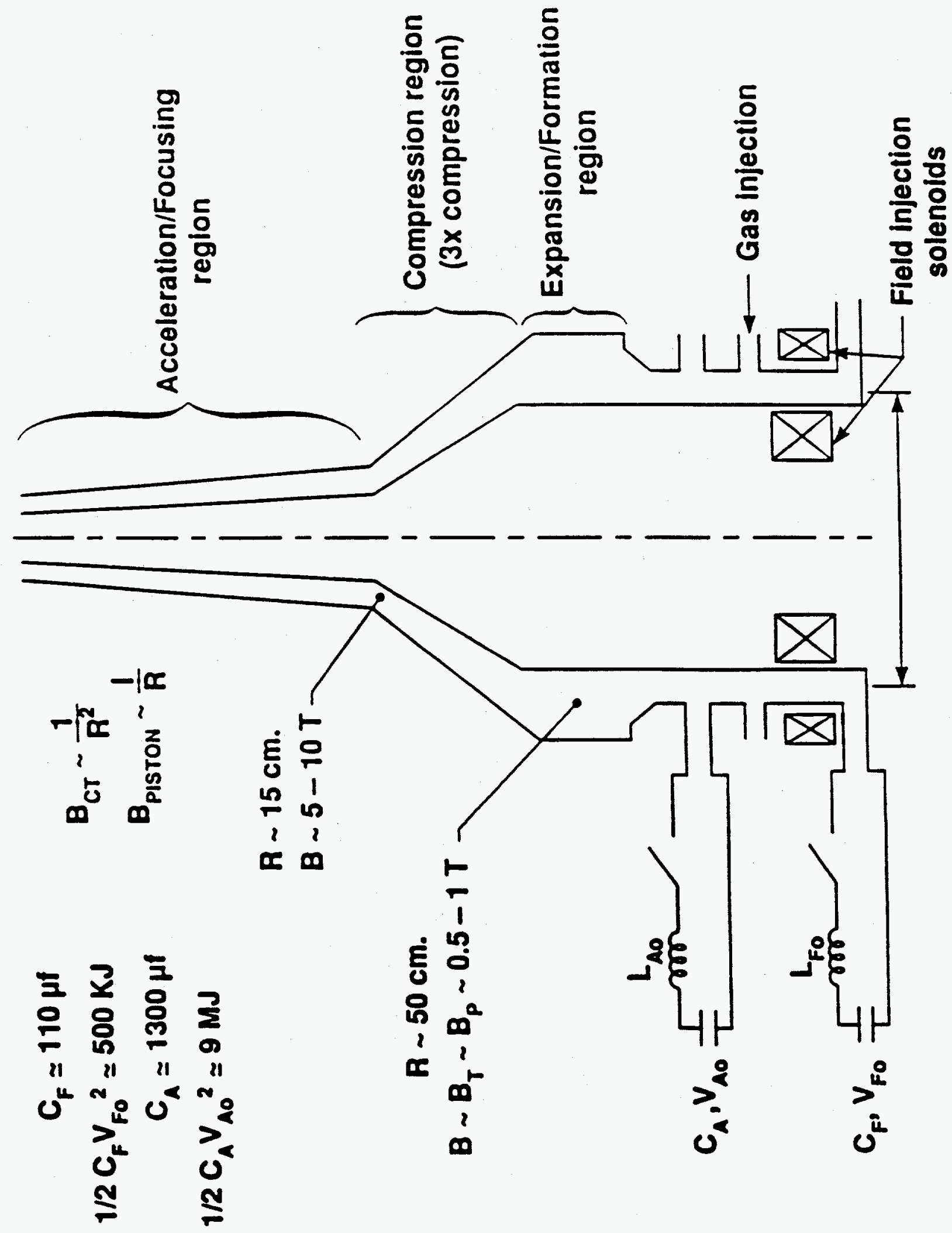

in 


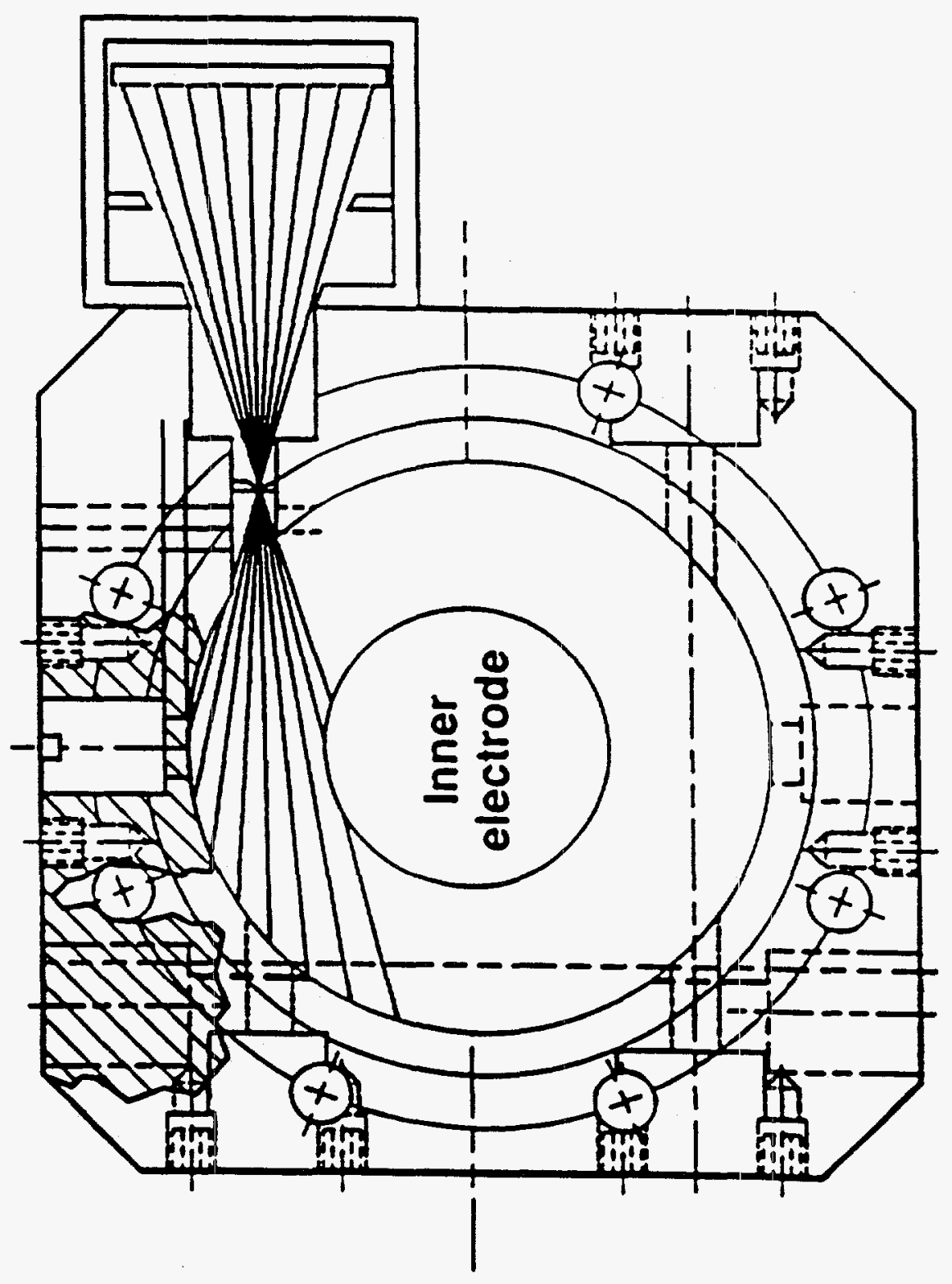

$\stackrel{n}{i}$ 


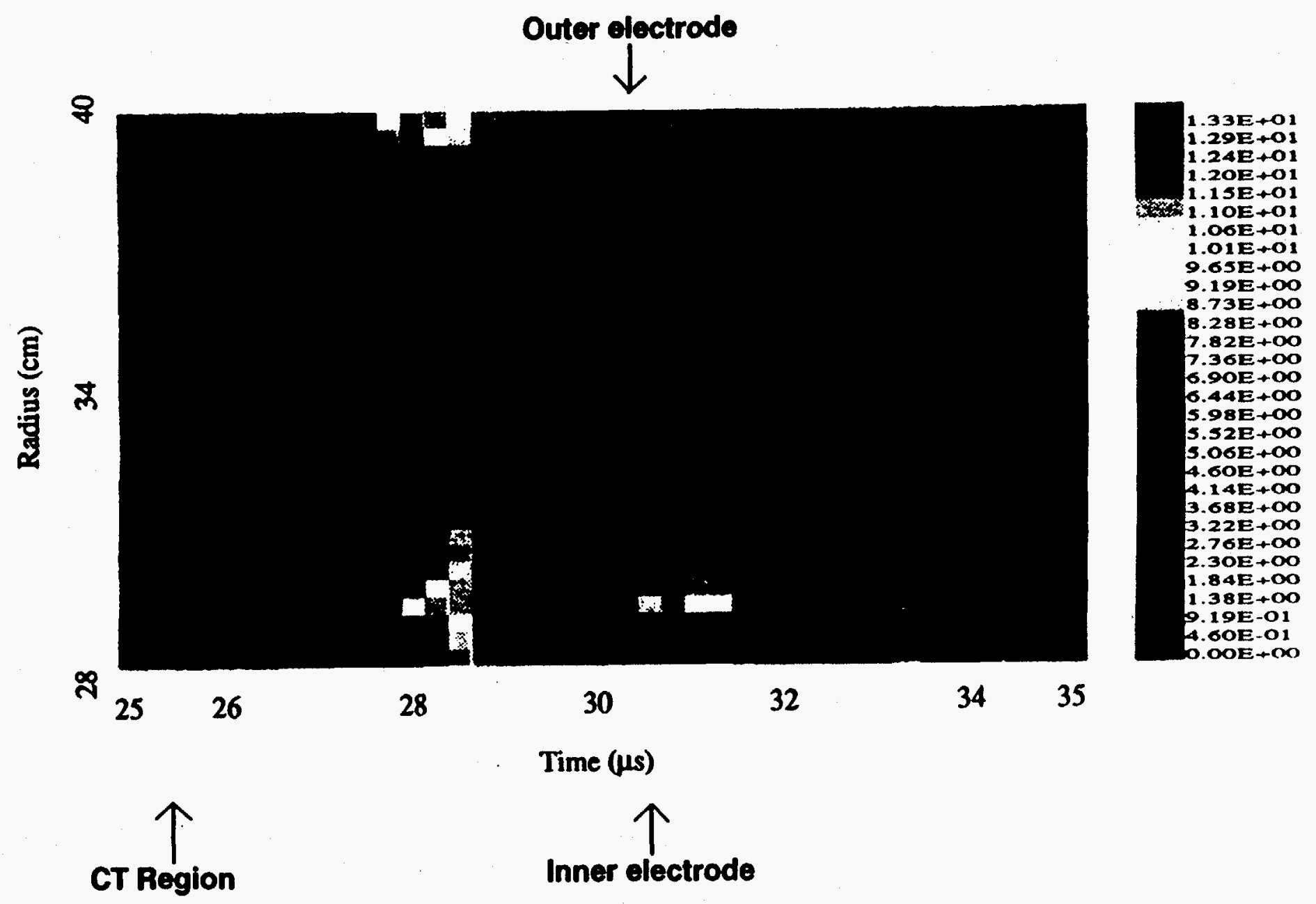

Fig. 3.3 


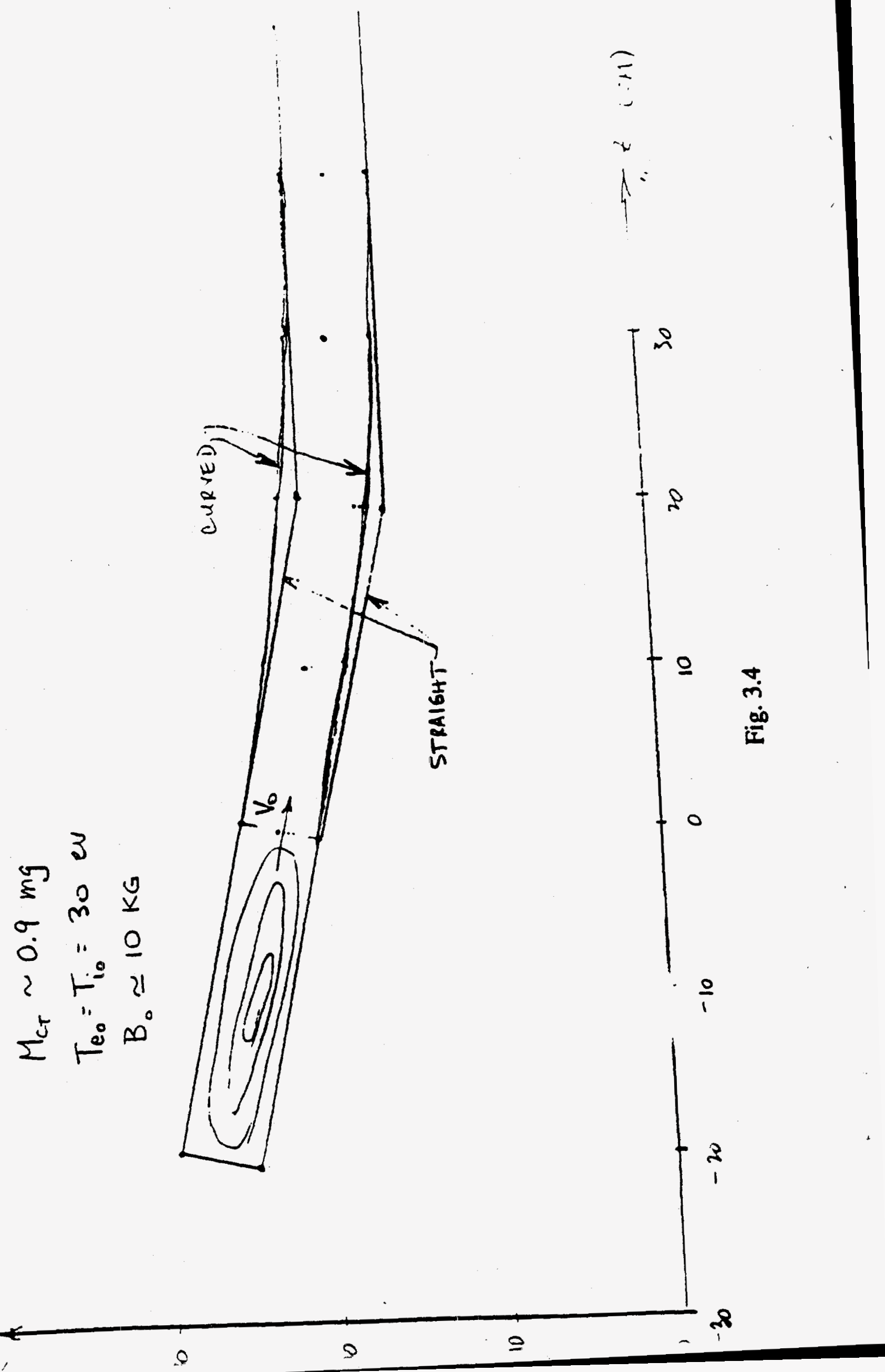


Radiated Energy (kJ)

KE (MJ) V(cm/us) Comp. Species $\leq 1 \mathrm{keV} 1-5 \mathrm{keV}$ 5-15 keV $\geq 30 \mathrm{keV}$

MARAUDER

$\begin{array}{rrrrrrrr}0.05 & 30 & 9 & \mathrm{Ne} & 3.1 & 10.4 & 5 \times 10^{-5} & \sim 0 \\ 0.25 & 40 & 10 & \mathrm{Ar} & 98.0 & 22.0 & 4 \times 10^{-3} & \sim 0 \\ 0.40 & 50 & 10 & \mathrm{Ar} & 46.0 & 73.0 & 0.4 & \sim 0 \\ 1.60 & 90 & 10 & \mathrm{Kr} & 66.0 & 270 . & 14.0 & 6 \times 10^{-3}\end{array}$

New Facility

10.0

200

$30 \quad X e$

210. 630.970.

620.

Fig. 3.5 


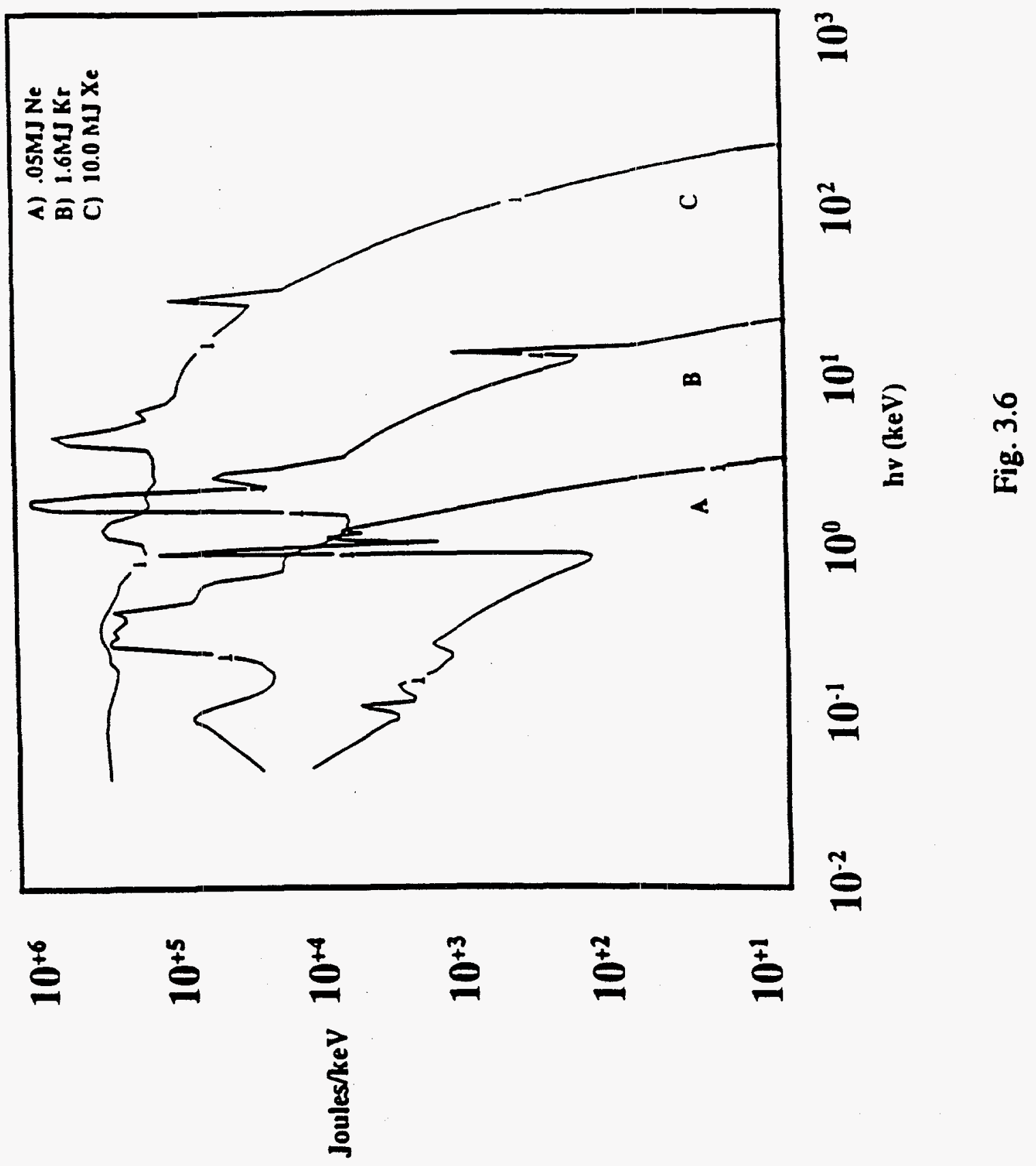


a) Circuit

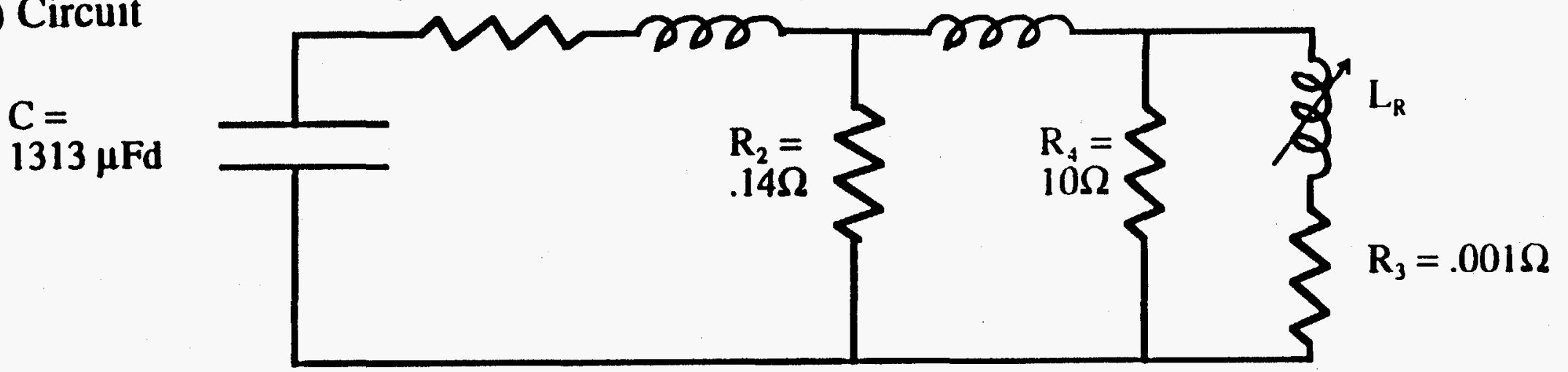

b) Geometry

Gap $=10 \mathrm{~cm}$

Fig. 4.1

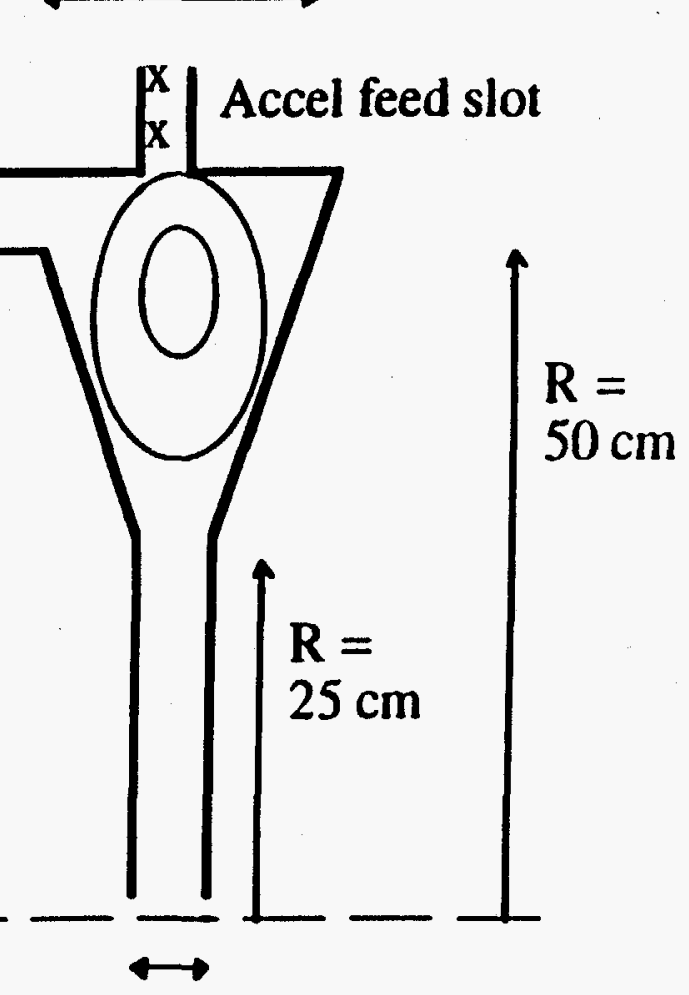

Gap $=2 \mathrm{~cm}$, constant for $\mathrm{R}<25 \mathrm{~cm}$ 


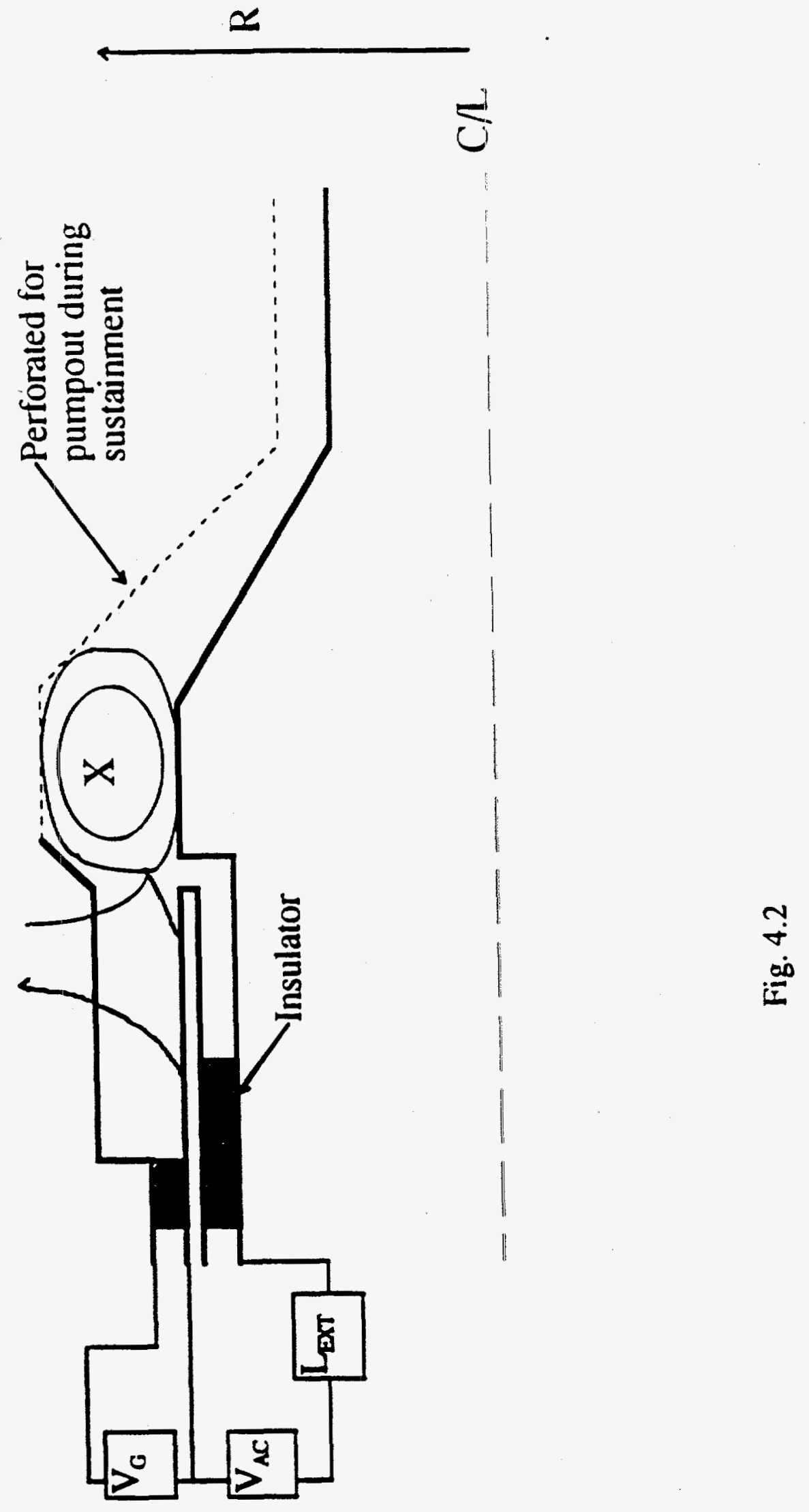




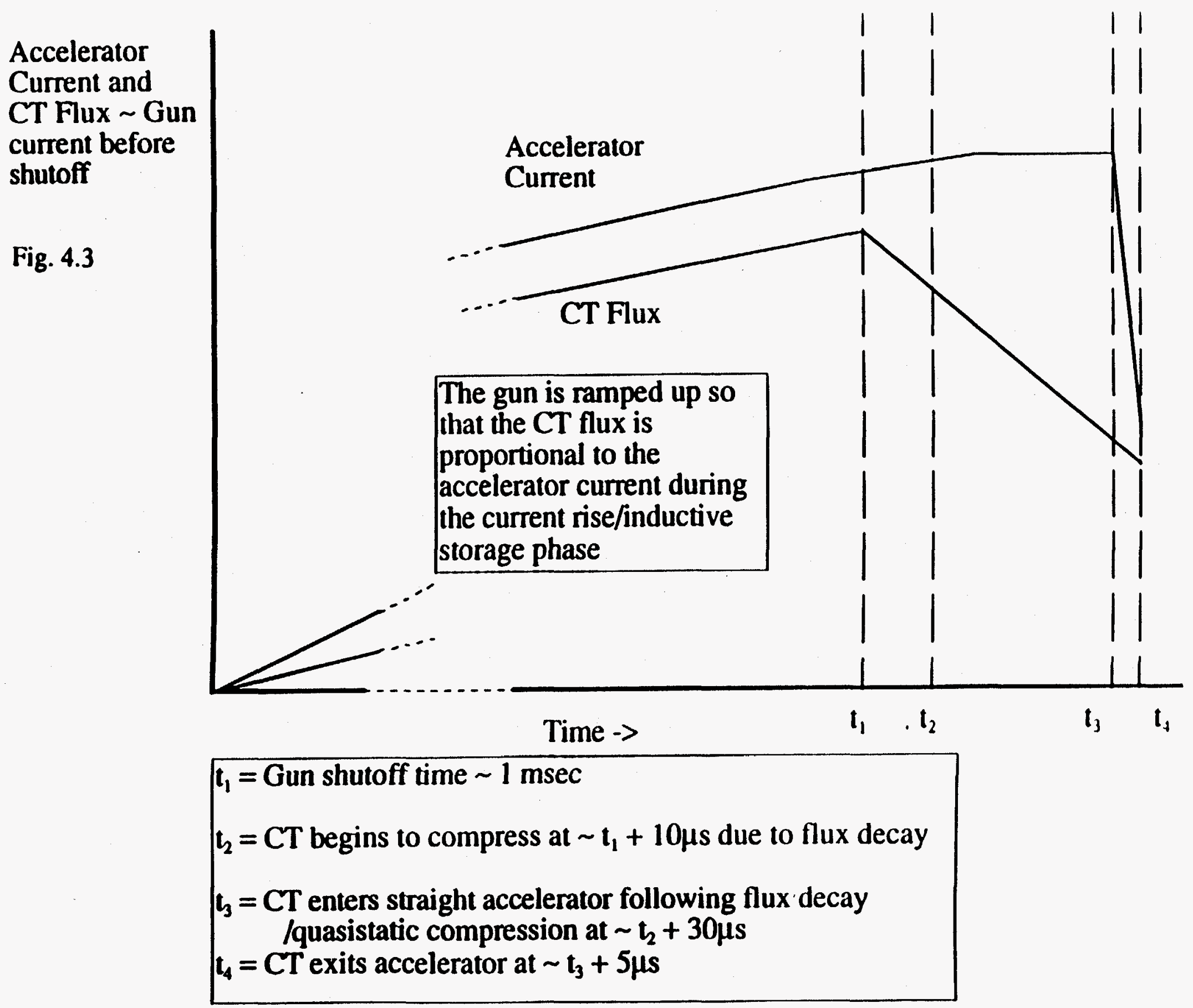

